\title{
Disentangling the Roles of RIM and Munc13 in Synaptic Vesicle Localization and Neurotransmission
}

\author{
${ }^{\circledR}$ Fereshteh Zarebidaki, ${ }^{\circledR}$ Marcial Camacho, ${ }^{\circledR}$ Marisa M. Brockmann, ${ }^{\circledR}$ Thorsten Trimbuch, \\ ${ }^{\circ}$ Melissa A. Herman, and ${ }^{\circledR}$ Christian Rosenmund \\ Institute of Neurophysiology and NeuroCure Cluster of Excellence, Charité-Universitätsmedizin, Berlin, 10117, Germany
}

Efficient neurotransmitter release at the presynaptic terminal requires docking of synaptic vesicles to the active zone membrane and formation of fusion-competent synaptic vesicles near voltage-gated $\mathrm{Ca}^{2+}$ channels. Rab3-interacting molecule (RIM) is a critical active zone organizer, as it recruits $\mathrm{Ca}^{2+}$ channels and activates synaptic vesicle docking and priming via Munc13-1. However, our knowledge about Munc13-independent contributions of RIM to active zone functions is limited. To identify the functions that are solely mediated by RIM, we used genetic manipulations to control RIM and Munc13-1 activity in cultured hippocampal neurons from mice of either sex and compared synaptic ultrastructure and neurotransmission. We found that RIM modulates synaptic vesicle localization in the proximity of the active zone membrane independent of Munc13-1. In another step, both RIM and Munc13 mediate synaptic vesicle docking and priming. In addition, while the activity of both RIM and Munc13-1 is required for $\mathrm{Ca}^{2+}$-evoked release, RIM uniquely controls neurotransmitter release efficiency. However, activity-dependent augmentation of synaptic vesicle pool size relies exclusively on the action of Munc13s. Based on our results, we extend previous findings and propose a refined model in which RIM and Munc13-1 act in overlapping and independent stages of synaptic vesicle localization and release.

Key words: active zone; electron microscopy; Munc13; RIM; synaptic transmission; synaptic vesicle

Significance Statement

The presynaptic active zone is composed of scaffolding proteins that functionally interact to localize synaptic vesicles to release sites, ensuring neurotransmission. Our current knowledge of the presynaptic active zone function relies on structurefunction analysis, which has provided detailed information on the network of interactions and the impact of active zone proteins. Yet, the hierarchical, redundant, or independent cooperation of each active zone protein to synapse functions is not fully understood. Rab3-interacting molecule and Munc13 are the two key functionally interacting active zone proteins. Here, we dissected the distinct actions of Rab3-interacting molecule and Munc13-1 from both ultrastructural and physiological aspects. Our findings provide a more detailed view of how these two presynaptic proteins orchestrate their functions to achieve synaptic transmission.

Received July 23, 2020; revised Sep. 22, 2020; accepted 0ct. 15, 2020.

Author contributions: C.R., F.Z., M.C., and M.M.B. designed research; C.R., F.Z., M.C., M.M.B., and M.A.H. edited the paper; F.Z. performed research; F.Z. analyzed data; F.Z. and M.C. wrote the first draft of the paper; F.Z. and M.C. wrote the paper; T.T. contributed unpublished reagents/analytic tools.

This work was supported by German Research Council Grant CRC 958 and Reinhart Koselleck project. We thank Berit Söhl-Kielczynski, Bettina Brokowski, Katja Pötschke, Sabine Lenz, Heike Lerch, Miriam Petzold, and Rike Dannenberg for excellent technical support; Charité Viral core facility for virus production and characterization; and Electron Microscopy and AMBI0 imaging core facilities at the Charité Campus Mitte for services.

The authors declare no competing financial interests.

Correspondence should be addressed to Christian Rosenmund at christian.rosenmund@charite.de. https://doi.org/10.1523/JNEUROSCI.1922-20.2020

Copyright $\odot 2020$ Zarebidaki et al.

This is an open-access article distributed under the terms of the Creative Commons Attribution 4.0 International license, which permits unrestricted use, distribution and reproduction in any medium provided that the original work is properly attributed.

\section{Introduction}

At presynaptic terminals, neurotransmitter release occurs at specific regions known as active zones (AZ). AZs are morphologically characterized by a complex network of proteins (Gundelfinger et al., 2003). The proteins that constitute the cytomatrix of the AZ (CAZ) play a central role in neurotransmitter release by localizing and clustering $\mathrm{Ca}^{2+}$ channels, and by regulating the stages of the synaptic vesicle (SV) cycle, such as tethering, docking, priming, and fusion (Sudhof, 2012). Among these functions, dissecting the role of specific molecular components in different steps of the SV cycle has presented technical challenges: (1) vesicle tethering and docking can only be distinguished from the downstream physiological events of vesicle priming and release by ultrastructure (Verhage and Sorensen, 2008); and (2) the individual protein components of the CAZ may contribute to more than one stage of neurotransmitter release (Ackermann et al., 2015). 
Because CAZ proteins are densely positioned at the AZ, with many putative interaction sites between them, disentangling the functions of individual $\mathrm{AZ}$ proteins requires a multitude of genetic ablation and rescue analyses. The AZ protein core consists of Rab3-interacting molecule (RIM), Munc13, RIM-binding protein (RIM-BP), ELKS, $\alpha$-Liprins, Piccolo, and Bassoon (Schoch and Gundelfinger, 2006; Sudhof, 2012). RIM is of particular interest, as investigations have shown its extensive interaction and regulatory effect on other AZ proteins. The two mammalian genes, Rims1 and Rims2, synthesize five RIM isoforms, $\operatorname{RIM} 1 \alpha / \beta$, and $\operatorname{RIM} 2 \alpha / \beta / \gamma$, whose domain content varies their interactions with $\mathrm{Rab3}$, Munc13, and $\mathrm{Ca}^{2+}$ channels (Kaeser et al., 2008, 2012). Genetic deletion of both Rim genes shows that $\alpha$ - and $\beta$-RIMs directly interact with $\mathrm{Ca}^{2+}$ channels, and indirectly, via RIM-BP, target $\mathrm{Ca}^{2+}$ channels to release sites, as the absence of RIMs leads to an impaired presynaptic $\mathrm{Ca}^{2+}$ channel function, $\mathrm{Ca}^{2+}$ channel-vesicle coupling, $\mathrm{Ca}^{2+}$-evoked release, and vesicular release probability (Hibino et al., 2002; Kiyonaka et al., 2007; Han et al., 2011, 2015; Kaeser et al., 2011; Graf et al., 2012; Hirano et al., 2017). In addition, $\alpha$-RIMs and RIM1 $\beta$ promote the activity of the priming factor, Munc13, by interfering with priming-impeding Munc13 homodimerization (Betz et al., 2001; Deng et al., 2011; Camacho et al., 2017) and by recruiting Munc13 to the presynaptic AZ (AndrewsZwilling et al., 2006). As Munc13s play a key role in determining the number of release sites and fusion-competent vesicles (Augustin et al., 1999a,b; Sakamoto et al., 2018; Bohme et al., 2019), it is challenging to deconvolve the direct role of RIM1/2 in the SV cycle from its role in stabilizing Munc13 at the AZ.

RIM also ensures the integrity of AZ scaffolds with its large molecular interactors, such as RIM-BP (Acuna et al., 2016; Wu et al., 2019) and ELKS (S. S. Wang et al., 2016). RIM's role in determining AZ scaffold integrity manifest on an ultrastructural level. Cryo-electron tomographic experiments revealed fewer tethered vesicles and a lower vesicle density in RIM1 $\alpha$ KO synaptosomes, suggesting a distinct role for RIM in vesicle localization close to the AZ membrane (Fernández-Busnadiego et al., 2013). This effect may be mediated by the dynamic tripartite complex of $\alpha$-RIMs, Rab3, and Munc13 that targets vesicles to the release sites (Dulubova et al., 2005). Therefore, comparative ultrastructural evidence in RIM and Munc13-deficient neurons is necessary to dissect the Munc13-dependent and -independent roles of RIM in SV localization and docking at the AZ.

In our study, we aimed to disentangle RIM's direct role in the SV cycle at the AZ from its role in enabling the function of Munc13. Using genetic deletions, we systematically compared murine hippocampal synapses lacking RIM1/2 and/or Munc13-1, the predominant isoform of Munc13 in mouse CNS. Comparative analysis of neurotransmission by electrophysiology, as well as ultrastructural analysis by electron microscopy, revealed a Munc131-independent contribution of RIM to vesicular release probability and SV localization. Together, our results provide a deeper understanding of key $\mathrm{AZ}$ proteins function individually and in concert to ensure efficient neurotransmission.

\section{Materials and Methods}

Animals and maintenance. All animal experiments and maintenance were approved by Animal Welfare Committee of CharitéUniversitätsmedizin Berlin and the Berlin state government agency for Health and Social Services (license no. T 0220/09). The RIMfflox/ $R I M 2^{\text {flox }}$ and $R B P 1^{\text {flox }} / R B P 2^{\text {flox }}$ mouse line, a gift from the Thomas C. Südhof laboratory (Acuna et al., 2016), was crossed with C57BL/6N mice to generate the RIM1 flox $/$ RIM $2^{\text {flox }}$ mouse line (called RIM $1 / 2^{\text {flox }}$ ).
The RIM1/2 $2^{\text {flox }}$ animals were interbred, and the offspring at postnatal (P) days $0-2$ were used to obtain the RIM1/2 control and conditional double KO (cDKO) neurons. Munc13-1 $1^{-1-}$ and Munc13-1 ${ }^{+/+}$littermates at embryonic (E) day 18 were obtained by interbreeding of Munc13- $1^{+/-}$mouse line on an FVB/N background. Here, we referred to Munc13-1 $1^{+/+}$as Munc13-1 WT and to Munc13-1 $1^{-1-}$ as Munc13-1 KO. Moreover, the mouse line of Munc13-2 $2^{-I-} /$ Muncl3-1 $^{+1-}$ on an FVB/ $\mathrm{N}$ background (Camacho et al., 2017) was interbred to produce Munc13-2 $2^{-/-}$Munc13-1 $1^{+/+}$animals at E18. Gender of the animals used for experimentation was not distinguished.

Neuronal cultures and lentiviral infections. Micro-islands and continental astrocyte feeder layers were generated from cortices of P0-P1 C57BL/6N mice 2 weeks before the neuronal culture preparations as described previously (Arancillo et al., 2013). To generate neuronal cultures, the hippocampi from either P0-P2 RIM1/2 flox pups, or Munc13-1 WT, Munc13-1 KO, Munc13-2 KO embryos at E18 were dissected. After enzymatic digestion with papain solution (Worthington), neurons were mechanically dissociated. Neurons were counted and seeded on astrocytic feeder layers in Neurobasal-A medium (Invitrogen) supplemented with B-27 (Invitrogen), $50 \mathrm{IU} / \mathrm{ml}$ penicillin, and $50 \mu \mathrm{g} / \mathrm{ml}$ streptomycin (Invitrogen). For autaptic cultures, 3500 cells were seeded on coverslip glasses $(30 \mathrm{~mm})$ containing an astrocytic micro-island pattern to perform immunocytochemistry and electrophysiological recordings. For the mass cultures, 100,000 cells were plated on 6-well plates with astrocytic feeder layers to perform Western blot, and 100,000 cells were seeded on sapphire glasses $(6 \mathrm{~mm})$ with an astrocytic feeder layer for high-pressure freezing experiments.

After 1-2 DIV, neurons were transduced with lentiviral particles. RIM1/2 control and cDKO were generated from RIM1/2 flox hippocampal neurons infected with lentivirus containing inactive and active Cre recombinase tagged with EGFP (Kaeser et al., 2011). Short hairpin RNA (shRNA) target sequence ( $5^{\prime}$-GCCTGAGATCTTCGAGCTTAT-3') for Munc13-1 was previously described (Deng et al., 2011). The shRNA sequence was cloned into a lentiviral shuttle vector that controlled its expression via a U6 promoter. A further human synapsin-1 promoter controlled the expression of an NLS-RFP protein to label infected neurons. The viral production was performed by the Viral Core Facility of the Charité-Universitätsmedizin Berlin. The titer of Munc13-1 KD shRNA was assessed by qPCR using LV900 Lentivirus Titration Kit (Applied Biological Materials).

Electrophysiology in the hippocampal autaptic culture. Whole-cell voltage-clamp recordings were performed on autaptic hippocampal neurons at DIV 15-20. Single neurons on micro-islands were selected and recorded at room temperature using Multiclamp 700B amplifier (Molecular Devices) under the control of Clampex 10.5 software. Axon Digidata 1550 digitizer (Axon Instruments) was used for data acquisition at $10 \mathrm{kHz}$ sample rate with a low-pass Bessel filter at $3 \mathrm{kHz}$. Borosilicate glass pipettes with resistance between 2 and $4 \mathrm{M} \Omega$ were pulled with a micropipette puller device (Sutter Instruments). The pipettes were filled with intracellular solution containing the following (in $\mathrm{mM}$ ): $136 \mathrm{KCl}$, 17.8 HEPES, 1 EGTA, $4.6 \mathrm{MgCl}_{2}, 4 \mathrm{Na}_{2} \mathrm{ATP}, 0.3 \mathrm{Na}_{2} \mathrm{GTP}, 12$ creatine phosphate, and $50 \mathrm{U} \mathrm{ml}^{-1}$ phosphocreatine kinase $(\sim 300 \mathrm{mOsm}, \mathrm{pH}$ 7.4). The extracellular solution contained the following (in $\mathrm{mm}$ ): 140 $\mathrm{NaCl}, 2.4 \mathrm{KCl}, 10 \mathrm{HEPES}, 10$ glucose, $2 \mathrm{CaCl}_{2}$, and $4 \mathrm{MgCl}_{2}(\sim 300$ mOsm, $\mathrm{pH}$ 7.4) and was permanently exchanged with a fast flow system. During the recordings, the access resistance was compensated at $70 \%$. The neurons with $<10 \mathrm{M} \Omega$ series resistance were used for the recordings. The EPSCs were induced by $2 \mathrm{~ms}$ depolarization from holding potential of -70 to $0 \mathrm{mV}$. Extracellular solution containing $3 \mu \mathrm{M}$ NBQX was used to distinguish glutamatergic from GABAergic neurons. To identify spontaneous events, traces recorded at a holding potential of $-70 \mathrm{mV}$ were filtered at $1 \mathrm{kHz}$, and mEPSCs were detected by a template algorithm in Axograph X (Axograph Scientific). False-positive events were excluded by subtracting events detected from traces in the presence of NBQX. The readily releasable pool (RRP) of SVs was estimated by application of $500 \mathrm{~mm}$ sucrose solution for $5 \mathrm{~s}$. The charge of the transient response component was used to determine the RRP size (Rosenmund and Stevens, 1996). The vesicular release probability $\left(P_{\mathrm{vr}}\right)$ was determined by dividing the EPSC charge by the sucrose-induced charge. 
Paired-pulse protocol was induced by two sequential action potentials (APs) with $25 \mathrm{~ms}$ interstimulus interval. Paired-pulse ratio was calculated by dividing the amplitude of the second EPSC by the amplitude of the first EPSC. The RRP augmentation was assessed by calculating the ratio of the sucrose-induced charge $2 \mathrm{~s}$ after $10 \mathrm{~Hz}$ stimulation with 50 APs to the baseline sucrose-induced charge. Electrophysiological recordings were analyzed using Axograph X (Axograph Scientific). Standard Hill equation $Y=M /\left[1+\left(K_{\mathrm{d}} / X\right)^{n}\right]$ was performed to fit the doseresponse relationship of electrophysiological parameters as a function of the protein expression (Arancillo et al., 2013). In the equation, $Y$ is response amplitude, $X$ is Munc13-1 relative expression to VGLUT1, $M$ is the maximum response, $K_{\mathrm{d}}$ is the dissociation constant, and $n$ is cooperativity.

High-pressure freezing and transmission electron microscopy. The 6 $\mathrm{mm}$ carbon-coated sapphire glass-containing neurons (DIV 15-16) were frozen in high-pressure freezing device (Leica Microsystems, EM ICE or HPM100). After freezing, the samples were transferred into a liquid nitrogen chamber. Each sample was moved inside the AFS2 automated freeze-substitution device (Leica Microsystems) containing cryovials for each sapphire with the following solution: $1 \%$ osmium tetroxide, $1 \%$ glutaraldehyde, $1 \% \mathrm{ddH}_{2} \mathrm{O}$ in anhydrous acetone. The AFS2 device was programmed for a $2 \mathrm{~d}$ protocol with a stepwise heating starting at $-90^{\circ}$ $\mathrm{C}$ for $4-5 \mathrm{~h},-90^{\circ} \mathrm{C}$ to $-20^{\circ} \mathrm{C}$ for $14 \mathrm{~h},-20^{\circ} \mathrm{C}$ for $12 \mathrm{~h}$, and $-20^{\circ} \mathrm{C}$ to $20^{\circ}$ $\mathrm{C}$ for $8 \mathrm{~h}$. Subsequently, samples were washed with anhydrous acetone and were treated for $1 \mathrm{~h}$ with $0.1 \%$ uranyl acetate to get contrast enhancement. In the last step, samples were embedded in EPON and baked at $60^{\circ} \mathrm{C}$ for $48 \mathrm{~h}$ to polymerize. The serial sectioning was performed with an ultramicrotome (Leica Microsystems) to obtain $40 \mathrm{~nm}$ sample thickness. Samples were collected in formvar-coated singleslot grids (Science Services). Just before the imaging, samples were contrasted for $3-5 \mathrm{~min}$ in $2 \%$ uranyl acetate and for $30 \mathrm{~s}$ in $0.3 \%$ lead citrate in $\mathrm{ddH}_{2} \mathrm{O}$. The images were obtained with a FEI Tecnai G20 transmission electron microscopy operating at $200 \mathrm{keV}$. Synapses were selected based on detectable postsynaptic density (PSD). For each experiment, $\sim 50$ profiles per group were imaged with (2048 $\times 2048$ pixels) CCD camera (Olympus) at $0.73 \mathrm{~nm}$ pixel size. The analysis was performed blind using ImageJ software and MATLAB. AZ length was defined as the membrane opposite to the PSD. SVs were identified as visible circular membranes structures with a diameter of $\sim 25-50 \mathrm{~nm}$ in a single plane of a $2 \mathrm{D}$ micrograph. The visualization of membranes was aided by increasing contrast. To avoid bias, the analysis for each experiment was performed by one person who was blind to the experimental groups. The SVs that were directly in contact with the AZ membrane were marked as docked SVs. SVs not directly in contact with the AZ membrane, but residing within $100 \mathrm{~nm}$ of the $\mathrm{AZ}$, were identified and their shortest distance to the AZ membrane was measured (Watanabe et al., 2013). To facilitate the presentation and discussion of our findings with regard to changes in SV localization as a function of RIM/Munc13-1 protein level manipulations, we used the term "proximal" to refer to the SVs up to $20 \mathrm{~nm}$ from the AZ membrane, whereas the SVs at $>20$ $100 \mathrm{~nm}$ from the AZ were referred to as "distal."

Immunostaining and quantification. Autaptic neurons at DIV 15-20 were fixed for $10 \mathrm{~min}$ in $4 \%$ PFA, permeabilized with $0.1 \%$ PBS-Tween20 solution and blocked with 5\% normal donkey serum. Rabbit antiVGLUT1 (SYSY 135302) and Guinea-pig anti-Munc13-1 (SYSY 126104) primary antibodies were used for coimmunostaining to detect Munc131 presynaptic expression. The primary antibodies were labeled with AlexaFluor-488 anti-rabbit IgG, as well as AlexaFluor-647 anti-guinea pig both in donkey serum (Jackson ImmunoResearch Laboratories), respectively. Single neurons on the astrocytic micro-islands were imaged using a Nikon scanning confocal microscopy A1Rsi with a $\times 60$ oil immersion objective. The $z$-series images at $0.3 \mu \mathrm{m}$ depth were obtained at equal exposure times with $1024 \times 1024$ pixels resolution and at the pixel size of $0.2 \mu \mathrm{m}$. The analysis was performed blind in ImageJ software by drawing ROIs of 50 synapses per neuron. ROIs were defined by staining for the SV marker VGLUT1. Munc13-1 fluorescence intensity for each synapse was divided to the corresponding fluorescence intensity of VGLUT1; $\sim 30$ neurons per group were analyzed in three independent cultures, and the data were normalized to the control of each experiment.

Western blot. Protein lysates for Western blot were obtained from mass culture neurons at DIV 15-20. The lysis solution contained $50 \mathrm{mM}$ Tris/HCl, pH 7.6, $150 \mathrm{mM} \mathrm{NaCl}, 1 \%$ Nonidet P-40, 0.5\% sodium deoxycholate, and $4 \%$ of Complete Protease Inhibitor (Roche Diagnostics). Lysed cells were scrubbed from plates and centrifuged to remove the debris from the protein-content supernatant; $30 \mu \mathrm{g}$ of proteins was loaded to SDS-polyacrylamide gel, separated after electrophoresis, and transferred to nitrocellulose paper (Bio-Rad). The proteins were labeled with primary antibodies during overnight incubation at $4^{\circ} \mathrm{C}$. The mouse antiTubulin III antibody (Sigma Millipore, T8660) was used to label Tubulin III as a loading control. Rabbit anti-Munc13-1 (SYSY 126103) or rabbit anti-RIM1/2 (SYSY 140203) was used to label Munc13-1 and RIM1/2, respectively. Goat IgG HRP-conjugated antibody (Jackson ImmunoResearch Laboratories) was used for the labeling of primary antibodies and was detected by ECL solution (GE Healthcare Biosciences). To quantify the proteins' expression level, the ratio of either Munc13-1 or RIM1/2 signal density to the corresponding Tubulin III signal density was normalized to the control group in three independent cultures.

Statistical analysis. All data were plotted with GraphPad Prism 7, and represented in bar plots as mean \pm SEM. First, the data were tested for normality with D'Agostino-Pearson test. If they did not pass the parametric assumption, Kruskal-Wallis test followed by Dunn's test was performed for multiple comparison, and Mann-Whitney $U$ test was applied for comparison of two unpaired datasets. In case the parametric assumption was passed, ordinary one-way ANOVA followed by Tukey's test for the multiple comparison, and Student's $t$ test for two datasets were applied. The $\alpha$ level was set at 0.05 . In the manuscript, the absolute values are shown as mean \pm SEM, and $n$ is the number of data points/ number of cultures. The overview statistics including sample size, mean, SEM, $p$ value, and result statistics for each figure are shown in Extended Data Figure 1-1 to Figure 7-1.

\section{Results}

\section{RIM1/2 and Munc13-1 differentially contribute to SV distribution at the $\mathrm{AZ}$}

In order to investigate the expression levels of RIM1/2 and Munc13-1 in our experimental system, we generated RIM1/2 control and cDKO neurons by transducing continental hippocampal cultures from RIM1/2 $2^{\text {flox }}$ mice (Kaeser et al., 2011) with either inactivated Cre-recombinase $(\Delta \mathrm{Cre})$ or Cre-recombinase (Cre), respectively. After 14 DIVs, we assessed protein expression of RIM1/2 and Munc13-1 in RIM1/2 control ( $\Delta$ Cre) and RIM1/ 2 cDKO (Cre) using Western blot analysis. We found that Cre expression in RIM1/2 ${ }^{\text {flox }}$ neurons leads to undetectable RIM1/2 levels, verifying successful removal of RIM1/2 proteins (Fig. 1A, left). Consistent with the previous reports (Deng et al., 2011), loss of RIM1/2 also resulted in severely reduced Munc13-1 protein levels (Fig. $1 A$, right), confirming the necessity of RIM in recruiting and stabilizing Munc13-1 at the AZ (AndrewsZwilling et al., 2006).

To identify the specific effects of RIM1/2 and Munc13-1 on synapse ultrastructure, we cryo-preserved cultures derived from Munc13-1 $1^{-1-}$ and Munc13- $1^{+/+}$neurons and RIM1/2 cDKO and control neurons under high pressure. We imaged $40 \mathrm{~nm} \mathrm{sec}-$ tions of synaptic profiles at high magnification in transmission electron microscopy (TEM) (Fig. 1C) and analyzed standard ultrastructure parameters, including PSD length, number of SVs docked at the AZ membrane (defined as the membrane opposite to the PSD), and the shortest distance of undocked vesicles to the AZ membrane (Fig. 1B). The AZ length was not different between the KO groups and their controls (Fig. $1 F, G$; RIM1/2 $\Delta$ Cre: $281.8 \pm 12.8 \mathrm{~nm}, n=153 / 3$; RIM1/2 Cre: $286.7 \pm 8.95 \mathrm{~nm}$, $n=161 / 3, p=0.094$; Munc13-1 WT: $312.3 \pm 10.72 \mathrm{~nm}, n=148 / 3$; 


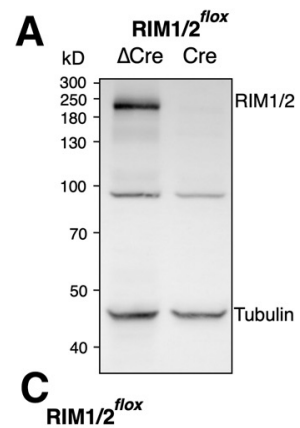

control ( $\triangle$ Cre )

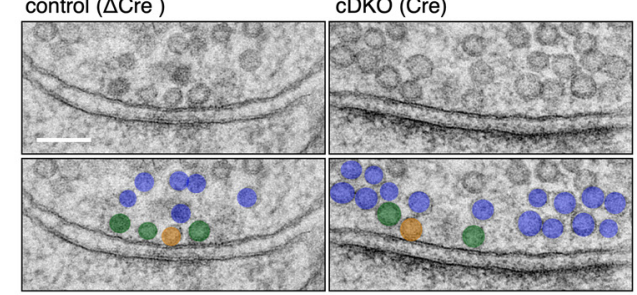

D

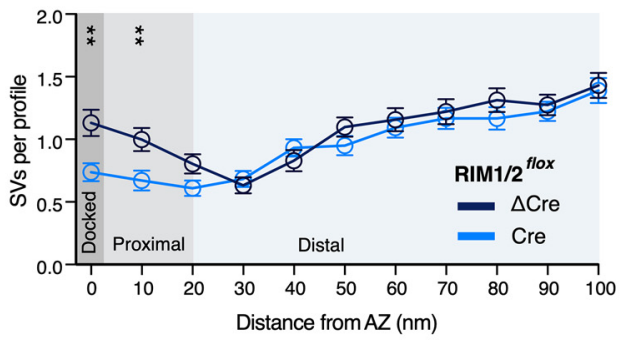

$\mathbf{F}$

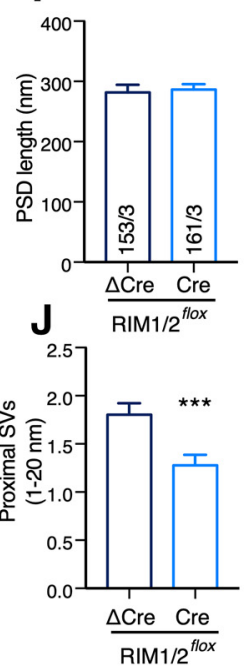

RIM1/2 ${ }^{\text {flox }}$

$\Delta$ Cre $\mathrm{Cre}$

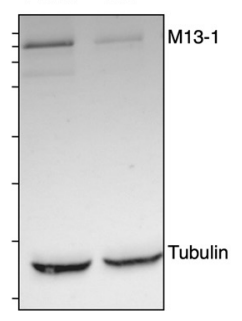

cDKO (Cre)

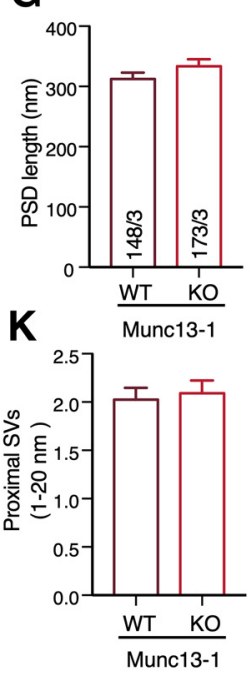

B
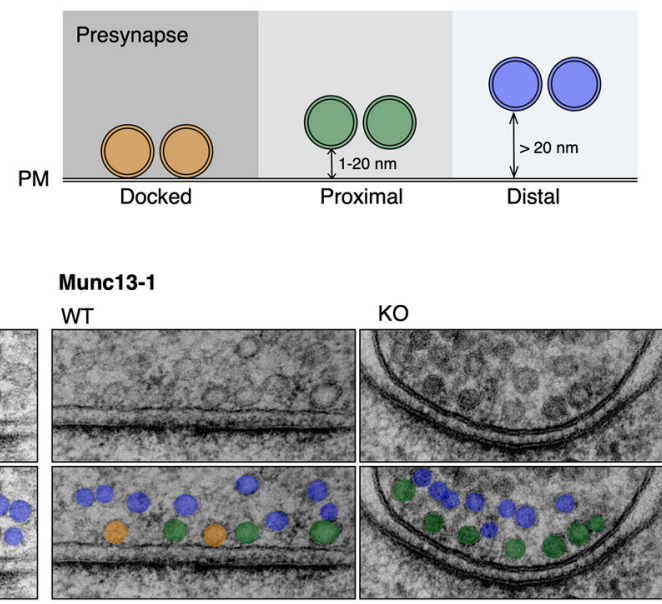

E

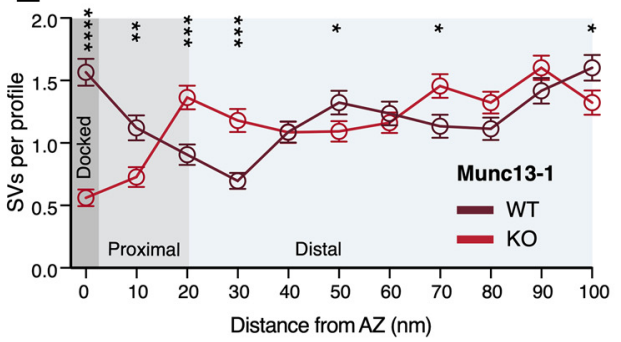

H

I

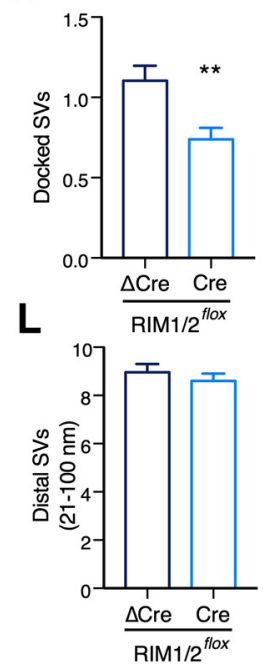

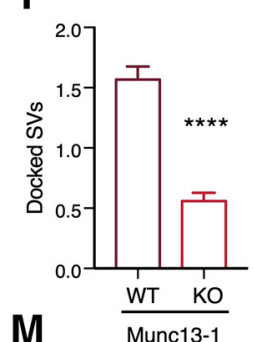

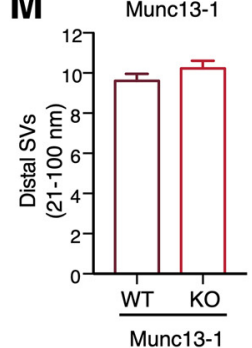

Figure 1. RIM1/2 and Munc13-1 differentially affect SV distribution and docking. $\boldsymbol{A}$, Immunoblots of lysates from RIM1/2flox hippocampal neurons infected with $\Delta$ Cre (control) and Cre recombinase (CDKO) detecting RIM1/2 (left) and Munc13-1 (right) expression. Tubulin expression was used as a loading control. Left, Markers designate the molecular weight. $\boldsymbol{B}$, Diagram represents the analysis of SVs based on their distance from AZ membrane. P.M., plasma membrane. C, Example TEM images displaying the presynaptic area of RIM1/2 control and CDKO (left), as well as Munc13-1 WT and KO (right). Top, Raw images. Bottom, Vesicles are color-coded according to their distance to the AZ membrane: docked SVs (orange), proximal SVs (green), and distal SVs (blue). Scale bar, $100 \mathrm{~nm}$. Additional example pictures are represented in Extended Data Figure 1-2. D, E, Plots represent the number of SVs as a function of distance from the AZ membrane for RIM1/2 CDKO (D) and Munc13-1 KO (E) synapses, compared with their corresponding control (binned to $10 \mathrm{~nm})$. $\boldsymbol{F}-\boldsymbol{M}$, Bar plots represent the mean PSD length $(\boldsymbol{F}, \boldsymbol{G})$, docked SVs $(\boldsymbol{H}$, I), proximal SVs $(1-20 \mathrm{~nm})(J, K)$, and distal SVs $(21-100 \mathrm{~nm})(L, M)$ for RIM1/2 CDKO and Munc13-1 K0 synapses compared with their corresponding controls. The data are obtained from the same experimental settings for all the electron microscopy analysis (RIM1/2 control: 153/3 and RIM1/2 CDK0: 161/3; Munc13-1 WT: 148/3 and Munc13-1 K0: 173/3) indicated in $\boldsymbol{F}$ and $\boldsymbol{G}$. The numbers are obtained from three independent cultures. Values indicate mean \pm SEM. $* p \leq 0.05 ; * * p \leq 0.01 ; * * * p \leq 0.001 ; * * * * p \leq 0.0001$; nonparametric $t$ test, followed by MannWhitney test. For the statistical overview, see also the table in Extended Data Figure 1-1.

Munc13-1 KO: $333.4 \pm 12.03 \mathrm{~nm}, n=173 / 3, p=0.382$, MannWhitney test). RIM1/2 cDKO presynaptic terminals showed a $30 \%$ reduction in docked SVs per AZ compared with controls (Fig. $1 H$; RIM1/2 $\Delta$ Cre: $1.10 \pm 0.09, n=153 / 3$; RIM1/2 Cre:
$0.73 \pm 0.07, n=161 / 3, p=0.002$, Mann-Whitney test). On the other hand, Munc13-1 KO synapses displayed a $\sim 70 \%$ reduction in SV docking (Fig. 1I; Munc13-1 WT: $1.57 \pm 0.10, n=148 / 3$; Munc13-1 KO: $0.56 \pm 0.07, n=173 / 3, p<0.0001$, Mann-Whitney 
test). Thus, high-pressure freezing and TEM experiments revealed SV docking deficits in cultures lacking either Munc13-1 or RIM1/ 2, albeit to a different extent.

Earlier studies have proposed a role for RIM in tethering SVs close to the AZ membrane (Fernández-Busnadiego et al., 2013). While we cannot assess tethers in our high-pressure frozen samples, we investigated the respective roles of RIM1/2 and Munc13-1 in the localization of nondocked SVs within $100 \mathrm{~nm}$ of the AZ membrane. RIM1/2 cDKO presynaptic terminals displayed a reduced number of SVs within the first $20 \mathrm{~nm}$ of the AZ membrane (Fig. $1 D$,j; RIM1/2 $\Delta$ Cre: $1.80 \pm 0.12, n=153 / 3$; RIM1/2 Cre: $1.28 \pm 0.10, n=161 / 3, p=0.0004$, Mann-Whitney test), but no change in density of SVs from $>20 \mathrm{~nm}$ up to $100 \mathrm{~nm}$ from the AZ membrane (Fig. $1 D, L$; RIM1/2 $\Delta$ Cre: $8.96 \pm 0.35, n=153 / 3 ; \mathrm{RIM} 1 / 2$ Cre: $8.60 \pm 0.3, n=161 / 3, p=$ 0.675 , Mann-Whitney test). Therefore, to facilitate the discussion about the relative SV localization, we defined $20 \mathrm{~nm}$ as a border to separate "proximal" SVs $(1-20 \mathrm{~nm})$ from the "distal" SVs (21-100 nm) to the AZ membrane (Fig. 1B).

On the other hand, Munc13-1-deficient presynaptic terminals showed no change in AZ-proximal SV number because of a significant SV accumulation near the AZ membrane (Fig. 1E, K; Munc13-1 WT: $2.03 \pm 0.12, n=148 / 3$; Munc13-1 KO: $2.09 \pm$ $0.13, n=173 / 3, p=0.853$, Mann-Whitney test). The different phenotype of two KOs in localization of SVs at the AZ-proximal region relative to their controls proposes that RIM localizes vesicles in the proximity to the AZ. Similar to RIM1/2 cDKO synapses, Munc13-1 KO synapses showed no change in distal SVs (Fig. 1E,M; Munc13-1 WT: $9.61 \pm 0.34, n=148 / 3$; Munc131 KO: $10.23 \pm 0.38, p=0.677, n=173 / 3$, Mann-Whitney test). Therefore, neither RIM1/2 nor Munc13-1 is specifically involved in distal SV localization.

\section{RIM1/2 localizes SVs in the AZ proximity independent of Munc13-1}

Since RIM1/2 deletion also results in a partial reduction of Munc13-1 protein levels (Deng et al., 2011), we aimed to separate RIM1/2 and Munc13-1 function by further reducing Munc13-1 protein in the RIM1/2 cDKO and control neurons using an shRNA knockdown (KD) approach (Fig. 2). Western blot analysis revealed a nearly complete abolishment of Munc13-1 protein in neuronal lysates infected with the shRNA (Fig. 2A). As expected, RIM1/2 proteins were diminished by $\sim 90 \%$ in RIM1/2 cDKO lysates (Fig. 2B). We also noted that KD of Munc13-1 in control neurons displayed a small but detectable reduction in RIM1/2 protein levels (Fig. 2B).

The KD of Munc13-1 in control neurons resulted in a decreased number of docked SVs compared with its control (Fig. 2F; RIM1/2 $\Delta$ Cre + Scramble: $2.13 \pm 0.11, n=157 / 3$; RIM1/2 $\Delta$ Cre + Munc13-1 KD: $1.42 \pm 0.09, n=148 / 3, p=0.0002$, Kruskal-Wallis test). While SV docking was reduced by $30 \%$ in RIM1/2 cDKO synapses, the additional KD of Munc13-1 in RIM1/2 cDKO caused a more severe docking impairment (Fig. 2F; RIM1/2 Cre + Scramble: $1.61 \pm 0.1, n=168 / 3$; RIM1/2 Cre + Munc13-1 KD: $0.95 \pm 0.07, n=167 / 3, p<0.0001$, KruskalWallis test). Hence, both RIM and Munc13-1 play a substantial role in SV docking. Moreover, while the total number of SVs within AZ-proximal region of Munc13-1 KD in control neurons did not alter (Fig. 2D,E,G), it decreased in RIM1/2 cDKO synapses compared with the control (Fig. 2D,E,G; RIM1/2 $\Delta$ Cre + Scramble: $2.53 \pm 0.13, n=157 / 3$; RIM1/2 $\Delta$ Cre + Munc13-1 KD: $2.95 \pm 0.18, n=148 / 3, p>0.999$; RIM1/2 Cre + Scramble: $1.95 \pm 0.13, n=168 / 3, p=0.002$, Kruskal-Wallis test). Interestingly,
Munc13-1 KD had no further effect on AZ-proximal SV number in RIM1/2 cDKO synapses (Fig. 2D,E,G; RIM1/2 Cre + Scramble: $1.95 \pm 0.13, n=168 / 3$, RIM1/2 Cre + Munc13-1 KD: $1.98 \pm 0.14$, $n=167 / 3, p>0.999$, Kruskal-Wallis test), illustrating a Munc13-1independent role of RIM in positioning SVs within close proximity to the AZ. In addition, the SV number in the AZ-distal region was not affected by the loss of RIM1/2, Munc13-1, or both (Fig. 2D,E,H; Kruskal-Wallis $(\mathrm{H})=1.92, p=0.587)$. Overall, our data demonstrate that RIM localizes SVs in proximity to the AZ in a Munc13-1-independent manner, while both RIM1/2 and Munc13-1 are required for SV docking.

\section{Deletion of RIM1/2 severely impairs SV priming and neurotransmitter release in glutamatergic hippocampal autaptic neurons}

Based on our findings that RIM1/2 influences SV docking and SV localization near the AZ membrane, we asked how RIM1/2 and Munc13-1 contribute to SV priming and neurotransmitter release in RIM1/2 or Munc13-1-deficient glutamatergic autaptic neurons. To examine how loss of RIM1/2 affects Munc13-1 protein levels at the synapse, we performed quantitative immunocytochemistry. By normalizing Munc13-1 immunofluorescence intensity to the corresponding signals from the SV marker VGLUT1 (Camacho et al., 2017), we found that Munc13-1 levels were reduced by $\sim 70 \%$ in the presynaptic terminals of autaptic RIM1/2 cDKO neurons (Fig. $3 B ; t_{(38)}=9.25, p<0.0001$, Student's $t$ test). This supports the notion that RIM1/2 stabilizes Munc13-1 at the AZ (Andrews-Zwilling et al., 2006).

To compare the roles of RIM and Munc13-1 in SV priming activity, we estimated the size of the RRP of SVs. RRP of RIM1/2 cDKO or Munc13-1 KO autaptic neurons and their respective controls was estimated by measuring the transient postsynaptic charge component evoked by the pulsed application of hypertonic sucrose (Rosenmund and Stevens, 1996) (Fig. 3C). Compared with the control neurons, RRP size was severely reduced by Munc13-1 deletion ( $95 \%)$ or RIM1/2 deletion $(\sim 88 \%)$ (Fig. $3 D$; RIM1/2 $\Delta$ Cre: $0.7 \pm 0.1 \mathrm{nC}, n=41 / 5$; RIM1/2 Cre: $0.08 \pm 0.01 \mathrm{nC}, n=51 / 5, p<0.0001$; Munc13-1 WT: $0.69 \pm 0.09 \mathrm{nC}, n=27 / 3$; Munc13-1 KO: $0.04 \pm 0.006 \mathrm{nC}, n=35$ / $3, p<0.0001$, Mann-Whitney test). Thus, the presence of RIM1/ 2 and Munc13-1 is required for efficient SV priming to occur, which goes in line with the SV docking role of RIM1/2 and Munc13-1 (Fig. 1). However, the impairment of SV priming was more drastic than the impairment in SV docking in both KOs.

To address the impact of RIM1/2 on $\mathrm{Ca}^{2+}$-evoked release in autaptic neurons, we analyzed the AP-evoked EPSCs (Fig. 3E). The EPSC amplitude in RIM1/2 cDKO autaptic neurons was reduced by $\sim 97 \%$, similar to the loss of EPSC amplitude observed in Munc13-1 KO neurons (Fig. 3F; RIM1/2 $\Delta$ Cre: $6.3 \pm 0.72 \mathrm{nA}, n=59 / 5 ; \mathrm{RIM} 1 / 2$ Cre: $0.18 \pm 0.05 \mathrm{nA}, n=56 / 5$, $p<0.0001$; Munc13-1 WT: 7.1 $\pm 0.89 \mathrm{nA}, n=32 / 3$; Munc13-1 KO: $0.19 \pm 0.03 \mathrm{nA}, n=46 / 3, p<0.0001$, Mann-Whitney test). While a large portion of the EPSC amplitude decrease in the absence of RIM1/2 is likely attributable to reduced SV priming activity, it is known that RIM1/2 also regulates $\mathrm{Ca}^{2+}$ channel recruitment (Han et al., 2011; Kaeser et al., 2011). We thus predicted that RIM1/2 cDKO autaptic neurons also had less efficacious $\mathrm{Ca}^{2+}$ secretion-coupling than Munc13-1 KO neurons, affecting vesicular release probability $\left(P_{\mathrm{vr}}\right)$. We computed the $P_{\mathrm{vr}}$ by calculating the ratio of the EPSC charge to the sucroseinduced charge. $P_{\mathrm{vr}}$ was decreased by $\sim 82 \%$ for RIM1/2 cDKO, 


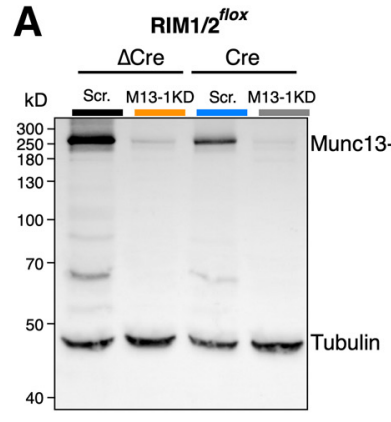

C

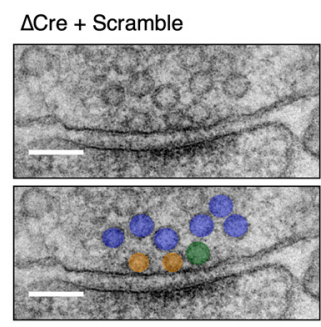

D

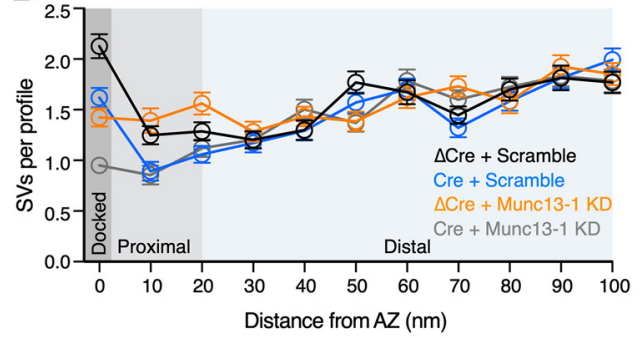

$\Delta \mathrm{Cre}+$ Munc13-1 KD
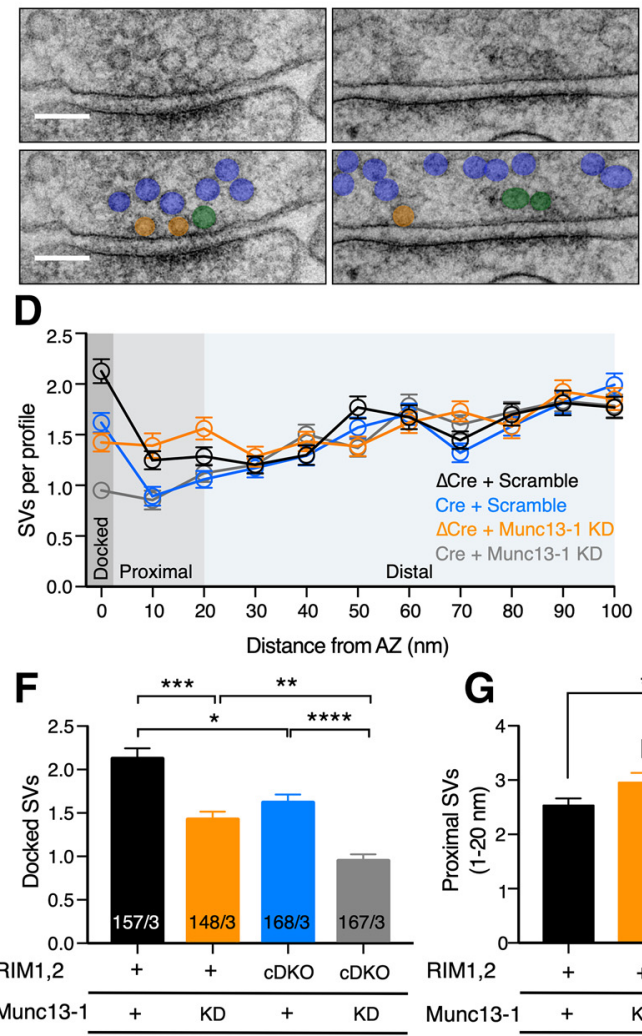

B $\quad$ RIM $1 / 2^{\text {ilox }}$
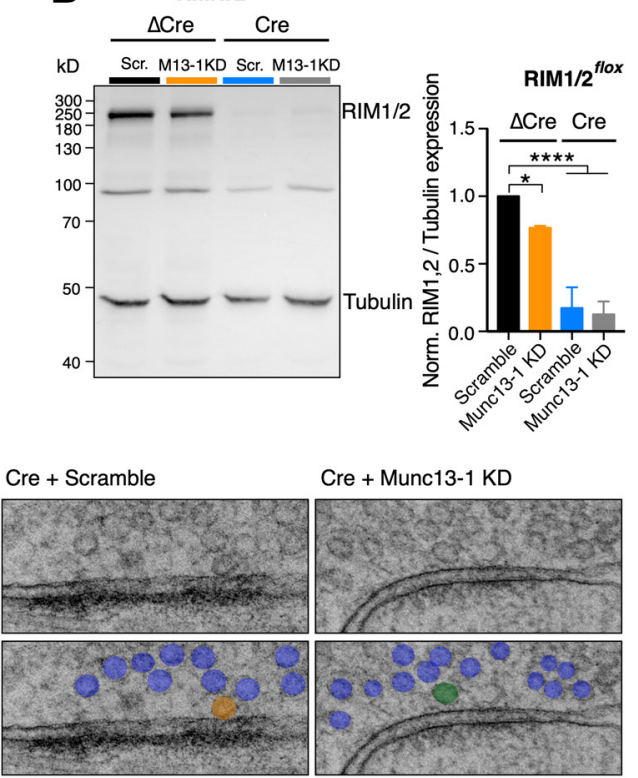

Cre + Munc13-1 KD

\section{E}

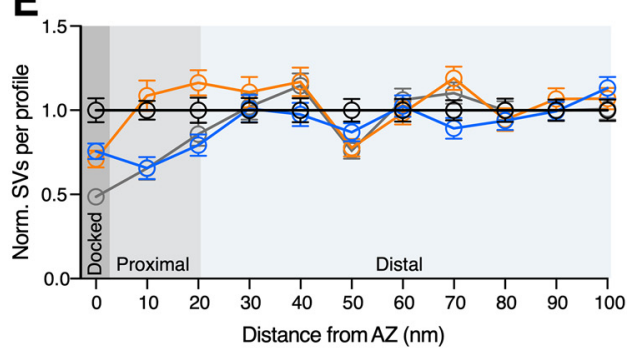

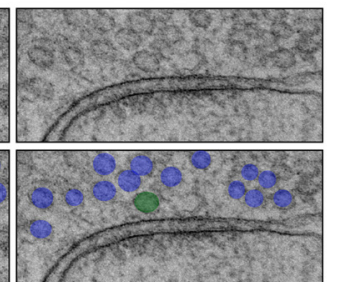

H

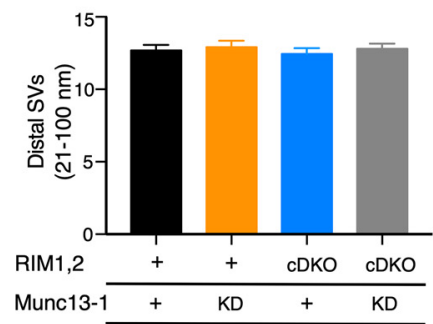

Figure 2. Munc13-1-independent impact of RIM on SV localization. $\boldsymbol{A}, \boldsymbol{B}$, Immunoblots (left) and bar plots (right) represent the expression of Munc13-1 ( $(\boldsymbol{A})$ and RIM1/2 (B) in RIM1/2flox cultures infected with $\Delta$ (re + Scramble (Scr.) shRNA (black), $\Delta$ Cre $+40 \times 10^{5}$ infectious units (IU) Munc13-1 KD shRNA (orange), Cre + Scramble shRNA (blue), and Cre $+40 \times 10^{5}$ Munc131 shRNA (gray). Tubulin expression was used as a loading control. Molecular weight markers are indicated on blots. The analysis was performed from three independent cultures. Significance was calculated using one-way ANOVA followed by Tukey's post hoc test $\left(\boldsymbol{A}: F_{(3,8)}=133.8, p<0.0001 ; \boldsymbol{B}: F_{(3,8)}=48.07, p<0.0001\right)$. C, Example TEM images of RIM1/2 flox hippocampal cultures infected as in $\boldsymbol{A}$ and $\boldsymbol{B}$. Top, Raw images. Bottom, Vesicles are color-coded according to their distance to the AZ membrane: docked SVs (orange), proximal SVs (green), distal SVs (blue). Scale bar, $100 \mathrm{~nm}$. Additional example pictures are represented in Extended Data Figure 2-2. D, E, Plot represents the SV number as a function of distance from the AZ membrane (binned $10 \mathrm{~nm} ; \boldsymbol{D}$ ) and the same values normalized to the control (binned to $10 \mathrm{~nm} ; \boldsymbol{E})$. $\boldsymbol{F}-\boldsymbol{H}$, Bar plots displaying the mean number of docked SVs (F), proximal SVs (1-20 nm) $(\boldsymbol{G})$, and distal SVs (21-100 nm) (H). Bar graph label "+" indicates endogenous expression of RIM1/2 or Munc13-1. "KD" refers to Munc13-1 KD. "CDKO" refers to RIM1/2-deficient neurons. Number of synaptic profiles for $\boldsymbol{D}$ $\boldsymbol{H}$ are indicated in $\boldsymbol{F}$. Values indicate mean \pm SEM. $* p \leq 0.05 ; * * p \leq 0.01 ; * * * p \leq 0.001 ; * * * * p \leq 0.0001$; nonparametric one-way ANOVA with Kruskal-Wallis test followed by Dunn's post hoc test. For the statistical overview, see also the table in Extended Data Figure 2-1.

while the loss of Munc13-1 led to a reduction of $P_{\mathrm{vr}}$ by only $\sim 56 \%$ (Fig. $3 G$; RIM1/2 $\Delta$ Cre: $6.4 \pm 0.56 \%, n=41 / 5$; RIM1/2 Cre: $1.11 \pm 0.3 \%, n=50 / 5, p<0.0001$; Munc13-1 WT: $8.08 \pm$ $0.74 \%, n=27 / 3$; Munc13-1 KO: $3.50 \pm 0.63 \%, n=35 / 3, p<0.0001$, Mann-Whitney test). We independently probed the efficiency of release by recording postsynaptic responses to paired-pulse stimulation protocols. Consistent with our observations of $P_{\mathrm{vr}}$ changes, we noticed a strong facilitation in the RIM1/2-deficient neurons (Fig. $3 H$ ), which confirms the stark decrease in release probability. Munc13-1-deficient neurons, in line with the less drastic $P_{\mathrm{vr}}$ loss, showed a moderate increase in facilitation (Fig. $3 H$; RIM1/2 $\Delta$ Cre:
$1.13 \pm 0.05, n=57 / 5 ; \mathrm{RIM} 1 / 2$ Cre: $1.63 \pm 0.11, n=54 / 5, p<$ 0.0001; Munc13-1 WT: $1.13 \pm 0.06, n=32 / 3$; Munc13-1 KO: $1.34 \pm 0.06, n=43 / 3, p=0.01$, Mann-Whitney test).

We also examined the impact of RIM1/2 and Munc13-1 on spontaneous release by analyzing the frequency of mEPSCs (Fig. $3 I$ ). Consistent with the reduced priming function, loss of both RIM and Munc13-1 proteins strongly impaired mEPSC frequency (Fig. 3J; RIM1/2 $\Delta$ Cre: $6.67 \pm 0.72 \mathrm{~Hz}, n=48 / 5$; RIM1/2 Cre: $1.48 \pm 0.33 \mathrm{~Hz}, \quad n=49 / 5, \quad p<0.0001$; Munc13-1 WT: $8.62 \pm 0.92 \mathrm{~Hz}, n=31 / 3$; Munc13-1 KO: $0.72 \pm 0.23 \mathrm{~Hz}, n=42$ / $3, p<0.0001$, Mann-Whitney test). 
A

B

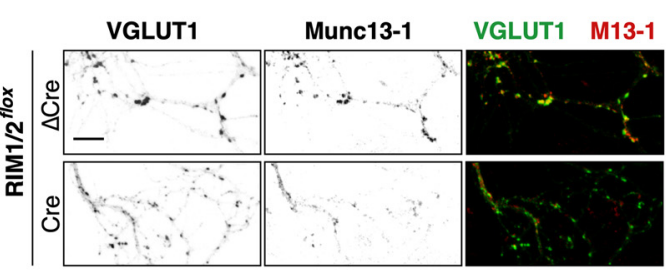

C

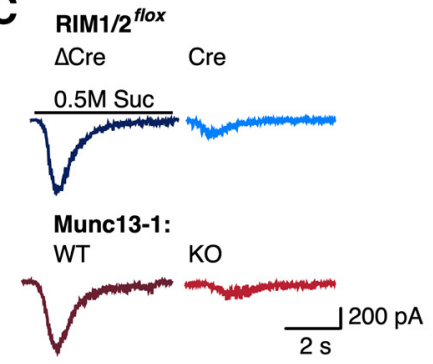

E
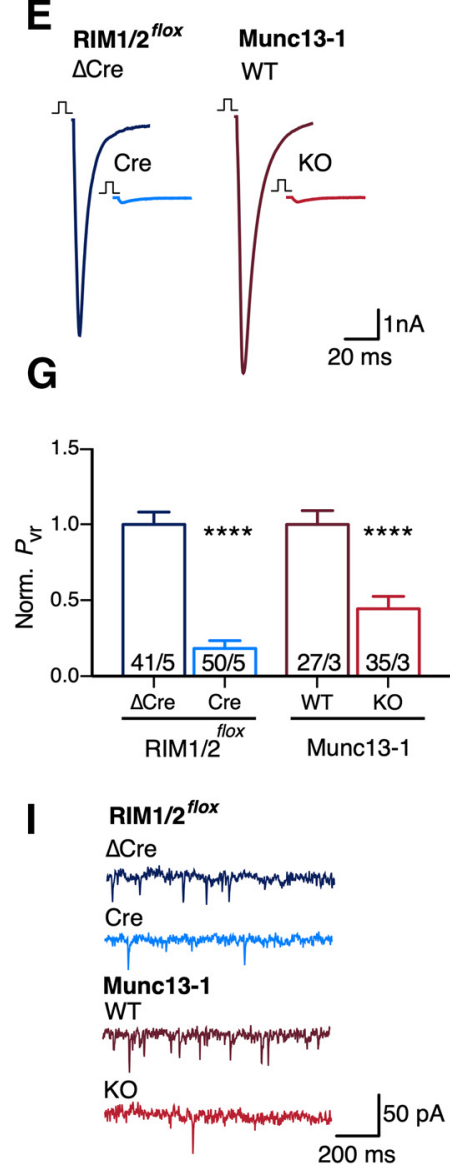

D

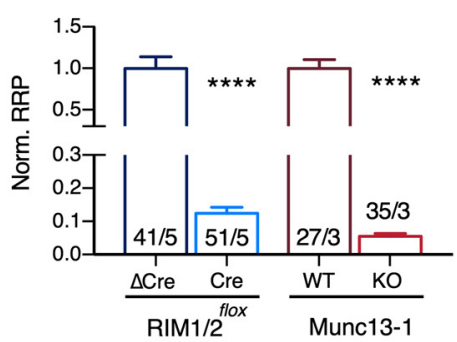

$\mathbf{F}$
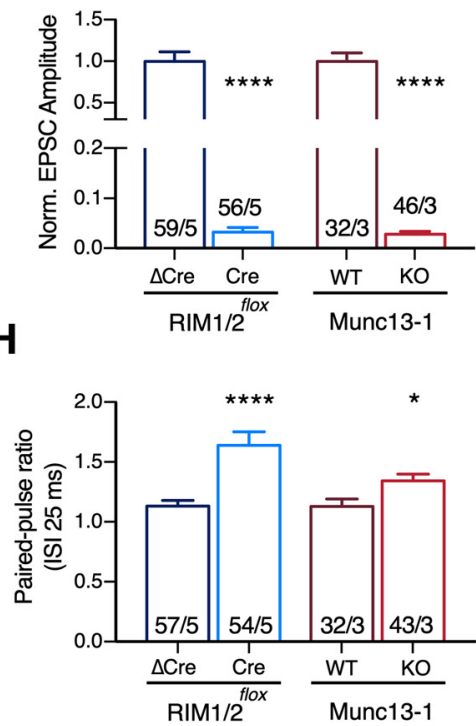

J

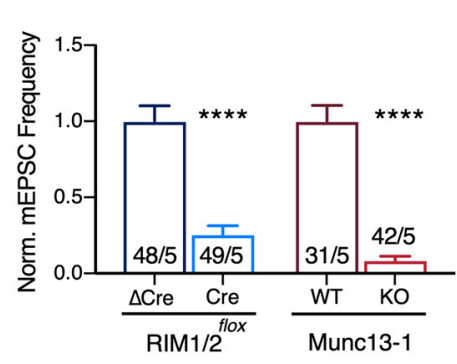

Figure 3. Comparison of synaptic properties of murine RIM1/2- and Munc13-1 KOs in autaptic hippocampal neurons. $\boldsymbol{A}$, Representative confocal microscopy projections display the immunofluorescence for Munc13-1 and VGLUT1 in RIM1/2 control and CDKO autaptic neurons. Scale bar, $10 \mu \mathrm{m}$. $\boldsymbol{B}$, Bar plot represents the ratiometric fluorescence intensity levels of Munc13-1 to VGLUT1 normalized to the control. Significance was calculated using Student's $t$ test $\left(t_{(38)}=9.25\right.$, $p<0.0001)$. C, Example traces of current induced by application of hypertonic solution (0.5 m sucrose [Suc], $5 \mathrm{~s}$ ) to estimate the size of RRP in neurons derived from RIM1/2 control (navy blue), RIM1/2 cDK0 (light blue), Munc13-1 WT (dark red), and Munc13-1 K0 (light red). D, Bar plots represent the normalized mean of RRP size (sucrose charge transfer). $\boldsymbol{E}$, Example traces of EPSCS from the same experimental groups as in $\boldsymbol{C}$. $\boldsymbol{F}$, Bar plot represents the normalized mean of EPSC amplitude. G, Bar plot represents the normalized vesicular release probability calculated by dividing the charge of EPSC to the sucrose charge. $\boldsymbol{H}$, Bar plot represents the paired-pulse ratio (with an interstimulus interval of $25 \mathrm{~ms}$ ). $\boldsymbol{I}$, Example traces of spontaneous release events from the same experimental groups as in $\boldsymbol{C}$. $\boldsymbol{J}$, Bar plot represents the normalized mean
These results confirm that both RIM1/2 and Munc13-1 are required for neurotransmission. While our data verify previous findings that both RIM1/2 and Munc13-1 regulate SV priming and release efficiency, we extend these findings by demonstrating the relative contributions of RIM1/2 and Munc13-1 to each of these processes. We found that RIM plays a major role in determining vesicular release probability and Munc13 predominantly controls vesicle priming.

\section{RIM1/2 cDKO loss of function does not} depend on Munc13-1 concentrations RIM influences Munc13-1 function by maintaining overall Munc13-1 protein levels in the synapse (Fig. $3 A$ ) and by activating Munc13-1 through disrupting the Munc131 homodimers (Deng et al., 2011; Camacho et al., 2017). To better understand the relative weight of these two functions of RIM and to gain a better mechanistic understanding of RIM's role in Munc13-1-mediated vesicle priming, we performed a graded shRNA-mediated KD of Munc13-1. To do so, cultures were infected with five doses of Munc13-1 shRNA ranging from 2 to $40 \times 10^{5}$ infectious units (IU). KD efficiency was assessed by both immunoblotting the hippocampal lysates (Fig. 4A) and immunocytochemistry on autaptic hippocampal cultures (Fig. 4C). Both analyses confirmed that by elevating the concentration of shRNA, Munc13-1 protein level was reduced in a dose-dependent manner, ranging from $70 \%$ to $95 \%$ (Fig. $4 B, D$ ).

We then proceeded by comparing RRP sizes between the groups and the control (Fig. 5A,B). Strikingly, despite similar Munc13-1 levels (30\% of control) in both RIM1/2 cDKO and lowest dose of Munc131 shRNA, the $2 \times 10^{5}$ IU Munc13-1 shRNA did not significantly alter the RRP size (Fig. $5 B$; RIM1/2 $\Delta$ Cre + Scramble: $0.42 \pm 0.05$ nC, $n=60 / 6$; RIM1/2 $\Delta$ Cre + Munc13-1 shRNA $2 \times 10^{5} \mathrm{IU}: \quad 0.40 \pm 0.06, \quad n=44 / 4$, $p>0.999$, Kruskal-Wallis test). Only at higher doses of shRNA, when Munc13-1 protein levels were reduced by $80 \%-95 \%$, the deficit in SV priming reached similar

$\leftarrow$

frequency of mEPSCs. All of the bar plots are normalized to the corresponding controls, except the paired-pulse ratio. Significances were calculated between the RIM1/2 control and CDKO (navy blue and light blue), and between Munc13-1 WT and $\mathrm{KO}$ (dark red and light red) using nonparametric $t$ tests, followed by a Mann-Whitney test. All numbers in bars indicate the cell number/culture number. Data indicate normalized mean \pm SEM. $* p \leq 0.05$. $* * * * p \leq 0.0001$. For the absolute values and statistical overview, see also the table in Extended Data Figure 3-1. 
A

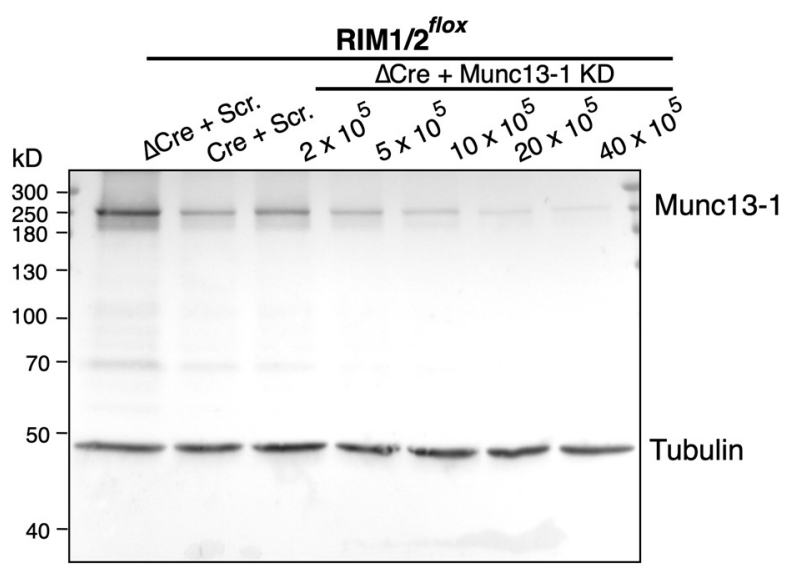

B
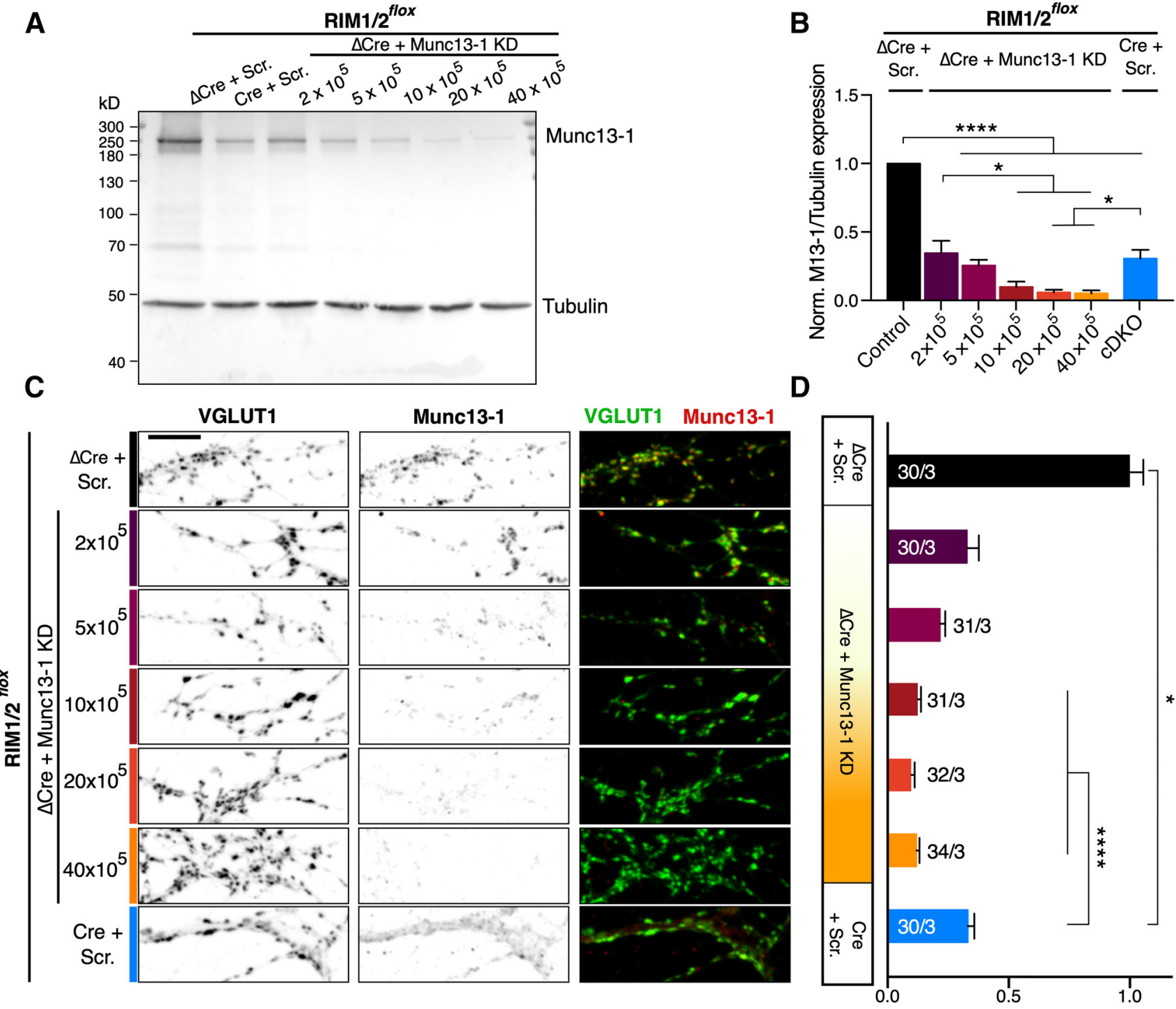

Norm. M13-1NGLUT1 flourescence intensity

Figure 4. Munc13-1 titration in hippocampal neurons. $A$, Immunoblot detecting Munc13-1 expression in RIM1/2fox lysates from cultured hippocampal neurons infected with $\Delta$ Cre + Scramble (Scr.) shRNA as control, $\Delta$ Cre + Munc13-1 KD shRNAs (2-40 $\times 10^{5}$ IU) to produce Munc13-1 dose gradients, and Cre recombinase + Scramble shRNA to create RIM1/2 cDKO. Tubulin expression was used as a loading control. Left, Molecular weight markers. B, Bar plot represents ratiometric quantification of Munc13-1 expression levels to tubulin. Data were collected from three independent cultures and normalized to the corresponding control. Significances were calculated using one-way ANOVA followed by Tukey's post hoc test $\left(F_{(6,14)}=46.79\right.$, $p<0.0001) . * p \leq 0.05 . * * * * \leq 0.0001$. C, Representative confocal microscopy projections display immunofluorescence intensity of Munc13-1 and VGLUT1 in the same experimental groups as in $\boldsymbol{A}$. Scale bar, $10 \mu \mathrm{m}$. D, Horizontal bar plot represents the ratiometric fluorescence intensity levels of Munc13-1 to VGLUT1 normalized to the control. For each neuron, $\sim 50$ synapses were measured and averaged. Statistical significances are represented only in comparison with RIM1/2 CDKO. Significances and $p$ values were calculated with a nonparametric one-way ANOVA with Kruskal-Wallis test followed by Dunn's post hoc test $(H=128.2, p<0.0001) . * p \leq 0.05 . * * * * p \leq 0.0001$. Data indicate normalized mean \pm SEM. For the statistical overview, see also the table in Extended Data Figure 4-1.

levels to RIM1/2 cDKO (Fig. 5B). Assuming that the role of RIM in priming is confined to Munc13-1 activation (Deng et al., 2011), our data indicate that RIM1/2 increases the effectiveness of Munc13-1 in SV priming by approximately fourfold.

When we compared the effect of graded Munc13-1 KD on evoked responses and spontaneous release events, we found that EPSC amplitudes and mEPSC frequency followed the reduction of RRP size (Fig. 5A-D). However, only with the KD of Munc13-1 protein level by $>90 \%$, the EPSC amplitude and mEPSC frequency reached the reduced level of the RIM1/2 cDKO neurons (Fig. 5C,D). Thus, RIM1/2 cDKO revealed the most severe phenotype in both parameters of EPSC amplitude and mEPSC frequency compared with the control.
In turn, $P_{\mathrm{vr}}$ was not majorly affected by Munc13-1 levels, whereas we observed a significant reduction in $P_{\mathrm{vr}}$ in RIM1/2 cDKO neurons (Fig. 5E). This suggests that reducing Munc13-1 levels does not impair $\mathrm{Ca}^{2+}$-secretion coupling, in contrast to the effect of eliminating RIM1/2.

By plotting SV priming and release as a function of Munc131 protein levels at the presynapse, we created dose-response plots of Munc13-1 for RRP size, EPSC amplitude, mEPSC frequency, and $P_{\mathrm{vr}}$ (Fig. 5F-I). We fitted the data from RRP size, EPSC amplitude, and mEPSC frequency measurements with a standard Hill equation (Arancillo et al., 2013) (see also Materials and Methods) to determine the relative sensitivity of synaptic function to Munc13-1 protein level and to define putative cooperativities. The fits show that the half-maximal SV priming, 
A
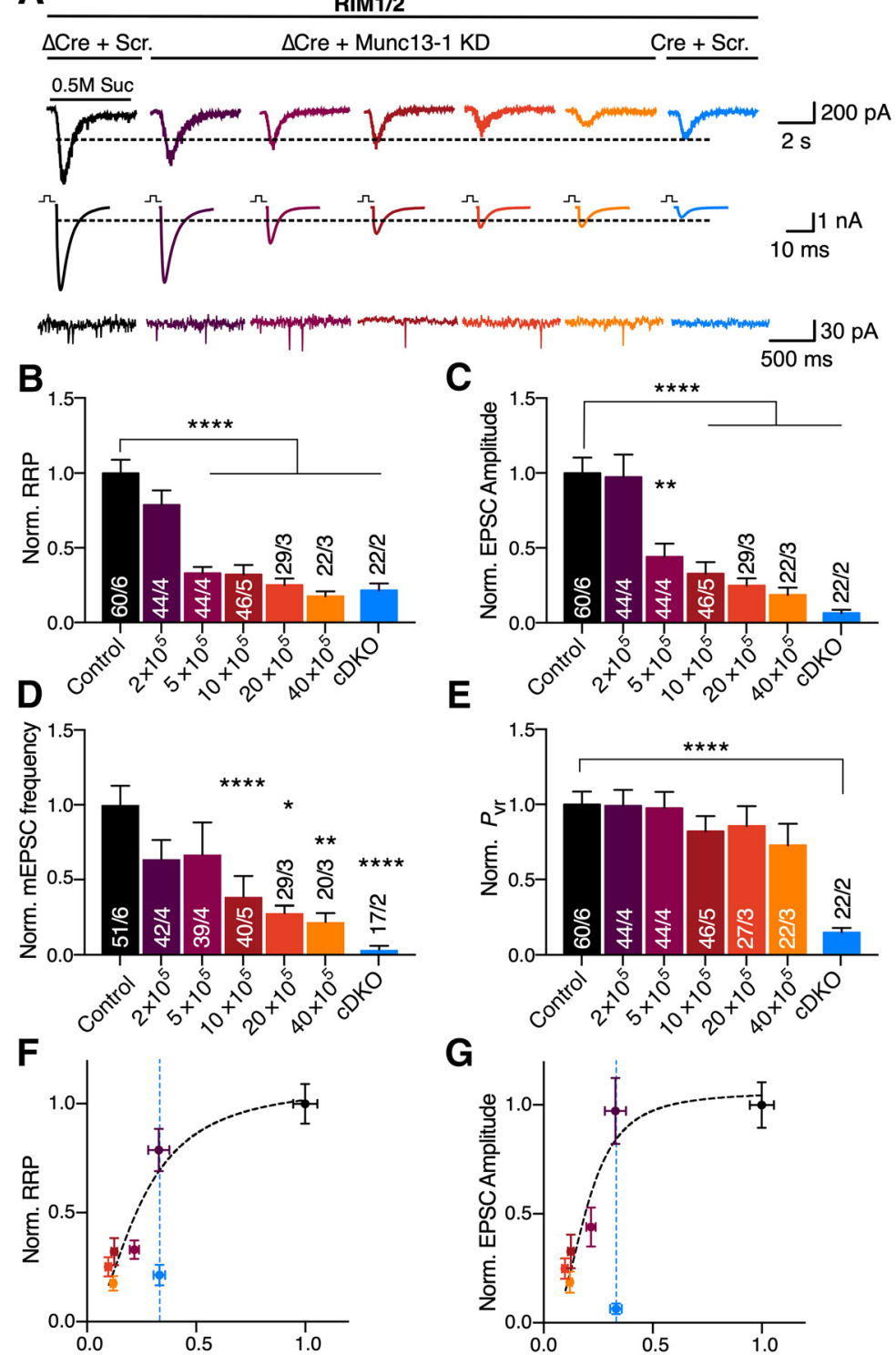

Norm. M13-1 presynaptic expression

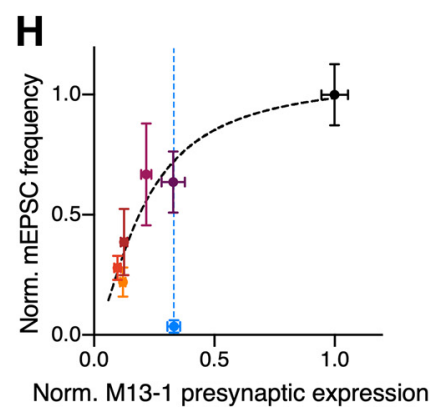

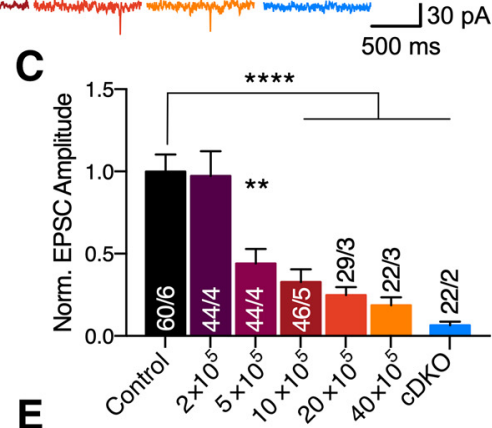

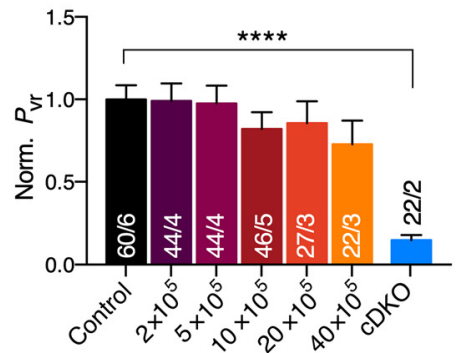

G

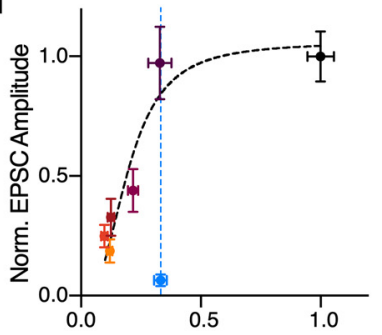

Norm. M13-1 presynaptic expression

\section{I}

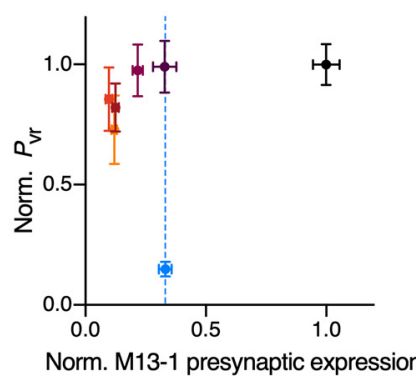

Figure 5. Quantification of RIM-dependent loss of Munc13-1 activity on synaptic properties. $A$, Sample traces of current induced by hypertonic sucrose to estimate the RRP size (top), sample traces of EPSCs (middle), and mEPSCs (bottom). Autaptic hippocampal neurons were infected with $\Delta$ Cre + Scramble (Scr.) shRNA as control, $\Delta$ Cre + Munc13-1 KD shRNAs $\left(2-40 \times 10^{5}\right.$ IU) to produce Munc13-1 dose gradients, and Cre recombinase + Scramble shRNA to create RIM1/2 CDKO. Dashed lines indicate the maximum current amplitude of RIM1/2 CDKO. $\boldsymbol{B}-\boldsymbol{E}$, Bar plots represent the normalized mean RRP defined as a charge measured by hypertonic sucrose application $(\boldsymbol{B})$, normalized EPSC amplitude $(\boldsymbol{C})$, normalized mEPSC frequency $(\boldsymbol{D})$, and normalized $P_{\mathrm{vr}}(\boldsymbol{E})$. $\boldsymbol{F}-\boldsymbol{I}$, Plots represent the normalized RRP size $(\boldsymbol{F})$, EPSC amplitude $(\boldsymbol{G})$, mEPSC frequency $(\boldsymbol{H})$, and $P_{\mathrm{vr}}(\boldsymbol{I})$ as a function of normalized Munc13-1/VGLUT1 presynaptic expression. $\boldsymbol{F}-\boldsymbol{I}$, Black dashed lines indicate fitting with the Hill equation. Blue dashed line indicates Munc13-1/VGLUT1 expression in RIM1/2 CDKO neurons. Data indicate normalized mean \pm SEM. $* p \leq 0.05 ; * * p \leq 0.01 ; * * * * p \leq 0.0001$; nonparametric one-way ANOVA

$\mathrm{Ca}^{2+}$-evoked release, and mEPSC frequency are achieved at $25 \%, 19 \%$, and $20 \%$ of WT Munc13-1 level, respectively (Fig. $5 F-H$ ). These processes follow positive cooperativity functions (1.9 for SV priming, 2.6 for $\mathrm{Ca}^{2+}$-evoked release, and 1.6 for mEPSC frequency). The similar sensitivity of Munc131 levels on the SV priming and mEPSC frequency functions suggests that the RRP is likely the source of vesicles released during spontaneous miniature events. These data also suggest that, at concentrations $<30 \%$ of WT levels, Munc13-1 is a rate-limiting factor for priming and release.

Additionally, we observed that the impairment in physiological measurements in RIM1/2 cDKO synapses (which express $\sim 30 \%$ of Munc13-1; Fig. 5F-I) did not follow the dose-response curves generated by Munc13-1 KD. This indicates that RIM1/2 cDKO loss of function does not only depend on Munc13-1 concentrations.

RIM1/2 controls $P_{\mathrm{vr}}$ independent from Munc13-1 but regulates SV docking and priming together with Munc13-1

To further assess the role of RIM independent of Munc13-1 function, we treated RIM1/2 control or deficient neurons with the highest dose Munc13-1 shRNA $\left(40 \times 10^{5} \mathrm{IU}\right)$. Nearcomplete removal of Munc13-1 in RIM1/2 cDKO neurons did not further reduce priming process and mEPSC frequency beyond the level of RIM1/2 cDKO alone (Fig. 6B; RIM1/2 Cre + Scramble: $0.12 \pm 0.03 \mathrm{nC}$, $n=61 / 5$; RIM1/2 Cre + Munc13-1 KD: $0.05 \pm 0.008 \mathrm{nC}, n=54 / 5, p=0.057$; Fig. $6 F$; RIM1/2 Cre + Scramble: $0.50 \pm 0.12 \mathrm{~Hz}$, $n=64 / 5 ;$ RIM1/2 Cre + Munc13-1 KD: $0.50 \pm 0.34 \mathrm{~Hz}, n=56 / 5, p>0.999$, KruskalWallis test), and showed a significant decrease compared with Munc13-1 KD in control neurons (Fig. $6 B$; RIM1/2 $\Delta$ Cre + Munc13-1 $\mathrm{KD}: \quad 0.08 \pm 0.009 \quad \mathrm{nC}, \quad n=50 / 5 ; \quad \mathrm{RIM} 1 / 2$ Cre + Munc13-1 KD: $0.05 \pm 0.008$ nC, $n=$ $54 / 5, p=0.015$; Fig. $6 F$; RIM1/2 $\Delta$ Cre + Munc13-1 KD: $\quad 1.07 \pm 0.22 \mathrm{~Hz}, \quad n=55 / 5$; RIM1/2 Cre + Munc13-1 KD: $0.50 \pm 0.34$ $\mathrm{Hz}, n=56 / 5, p=0.026$, Kruskal-Wallis test). These data provide supporting evidence for a function of RIM as an activator of Munc13-1 in SV priming (Deng et al., 2011; Camacho et al., 2017), and suggest that both RIM and Munc13-1 are required for priming function. Moreover, removal of Munc13-1 in the RIM1/2-deficient neurons did not further

$\leftarrow$

with Kruskal-Wallis test followed by Dunn's post hoc test. For the absolute values and statistical overview, see also the table in Extended Data Figure 5-1. 
A

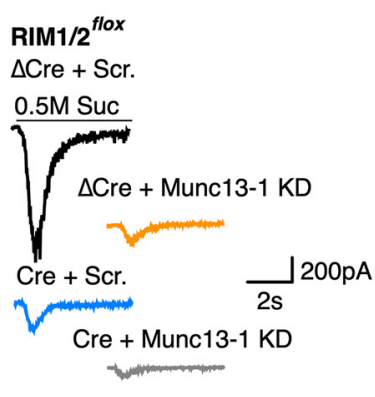

C

$$
\text { RIM1/2 } 2^{\text {IIox }}
$$

$\Delta \mathrm{Cre}+\mathrm{Scr}$.

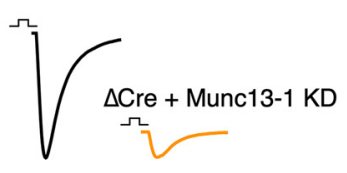

Cre + Scr.

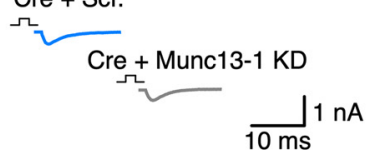

E

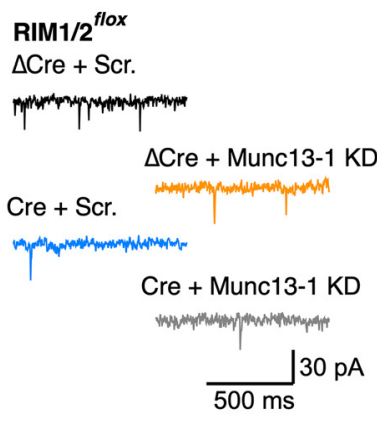

G

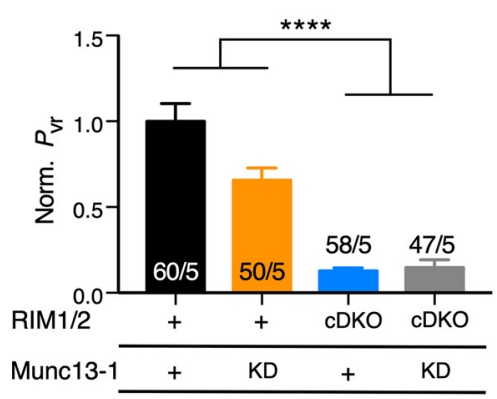

B
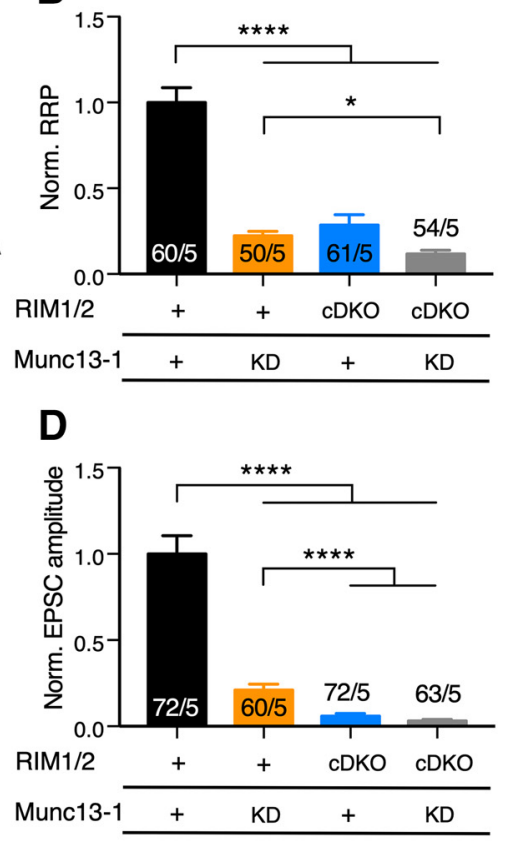

$\mathbf{F}$

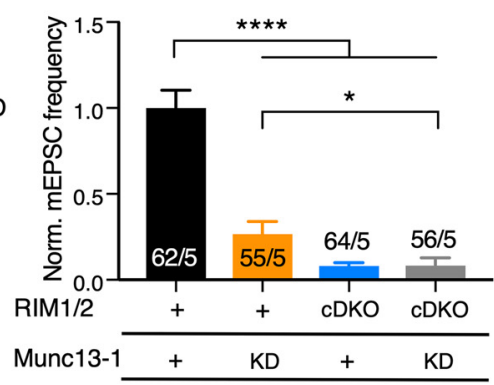

H

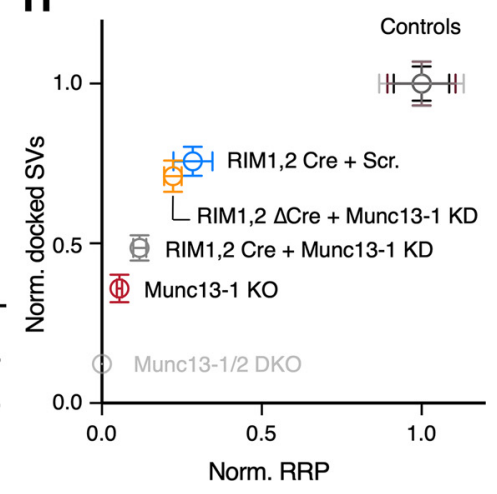

Figure 6. RIM-independent function of Munc13-1 on synaptic properties. $\boldsymbol{A}$, Sample traces represent the current evoked by hypertonic sucrose as an estimate for RRP in RIM1/2 flox cultures infected with $\Delta$ (re + Scramble (Scr.) shRNA (black), $\Delta$ (re $+40 \times 10^{5}$ IU of Munc13-1 KD shRNA (orange), Cre + Scramble shRNA (blue), and Cre $+40 \times 10^{5}$ Munc13-1 shRNA (gray). B, Bar plot represents the normalized mean of charge released by hypertonic sucrose (referred to as RRP). $\boldsymbol{C}$, $\boldsymbol{D}$, Sample traces of EPSC $(\boldsymbol{C})$ and bar plot of normalized EPSC amplitude. $\boldsymbol{E}, \boldsymbol{F}$, Sample traces of spontaneous release events $(\boldsymbol{E})$, and the bar plot represents the normalized mean mEPSC frequency $(\boldsymbol{F})$. $\mathbf{G}$, Bar plot represents the normalized $P_{\mathrm{vr}}$. Normalization in all of the bar plots is performed relative to the corresponding control ( $\Delta$ Cre + scramble; black). In graph labels, " + " refers to the endogenous expression. "KD" refers to the Munc13-1 KD. "CDKO" refers to RIM1/2 deficiency. Numbers in bars indicate the cell number/culture number. Data indicate normalized mean \pm SEM. $* p \leq 0.05$; $* * * * p \leq 0.0001$; nonparametric one-way ANOVA with Kruskal-Wallis test followed by Dunn's post hoc test. For the absolute values and statistical overview, see also the table in Extended Data Figure 6-1. H, Plot represents the SV docking/ priming relationship. Munc13-1/2 DKO data are adapted from Camacho et al. (2017). Controls represent $\Delta$ Cre + Scr., Munc13-1 WT, and Munc13-1/2 DK0 rescue with WT Munc13-1 (Camacho et al., 2017).

impair the EPSC amplitude (Fig. 6D; RIM1/2 Cre + Scramble: $0.23 \pm 0.06 \mathrm{nA}, n=72 / 5$; RIM1/2 Cre + Munc13-1 KD: $0.13 \pm$ $0.04 \mathrm{nA}, n=63 / 5, p=0.745$, Kruskal-Wallis test) and $P_{\mathrm{vr}}$ (Fig. 6G; RIM1/2 Cre + Scramble: $0.8 \pm 0.11 \%, n=58 / 5$; RIM $1 / 2$ Cre + Munc13-1 KD: $0.95 \pm 0.29 \%$, $n=47 / 5, p>0.999$, Kruskal-Wallis test), again emphasizing that RIM predominantly controls the $\mathrm{Ca}^{2+}$-triggered release and $P_{\mathrm{vr}}$.

Recent studies correlated SV docking to the priming process, where the loss of SV docking was accompanied by loss of SV priming (Siksou et al., 2009; Imig et al., 2014). As our molecular and genetic manipulations of RIM1/2 and Munc13-1 protein levels provided a range of SV docking and priming impairments, we used these data to obtain the levels of SV priming as a function of the SV docking activity. When plotting these two functions (Fig. $6 H$ ), we found that Munc13- and RIM-dependent docking and priming function did not show a linear correlation. Indeed, vesicle priming was more sensitive to protein levels than SV docking in all RIM and Munc13 KOs/KDs. This may indicate either a lower stoichiometry of RIM and Munc13 for SV docking than priming, or that the $2 \mathrm{D}$ ultrastructural image analysis has lower resolution for detecting an SV docking deficit. Furthermore, the effect on SV docking/priming observed in the absence of RIM illustrated a sensitivity defined by the reduction of Munc13-1 protein levels. This supports the notion that the role of RIM1/2 in SV docking and priming can be simply explained by its role in recruiting and activating Munc13-1, as previously proposed (Andrews-Zwilling et al., 2006; Deng et al., 2011; Camacho et al., 2017).

RIM1/2 does not contribute to activitydependent RRP augmentation

Our ultrastructural results suggested that RIM recruits SVs to the proximity of $\mathrm{AZ}$ membrane (Fig. 2); therefore, we investigated whether this function of RIM supplies SVs to the activity-dependent RRP augmentation that occurs in the absence of Munc13-1 (Rosenmund et al., 2002). To do so, we probed the RRP size by hypertonic sucrose application before and $2 \mathrm{~s}$ after $50 \mathrm{APs}$ at $10 \mathrm{~Hz}$ high-frequency stimulation (Rosenmund et al., 2002) (Fig. 7A). Munc13-1 KD in either control or RIM1/2 cDKO synapse exhibited an activity-dependent augmentation of RRP size (RRP ratio: Fig. $7 B$; RIM1/2 $\Delta$ Cre + Scramble: $0.89 \pm 0.03, n=31 / 4$; RIM1/2 $\Delta$ Cre + Munc13-1 KD: $1.33 \pm 0.08$, 
$n=28 / 4, \quad p<0.0001 ; \quad$ RIM1/2 Cre + Munc13-1 KD: $1.45 \pm 0.11, n=23 / 4$, $p<0.0001$, Kruskal-Wallis test). This suggests that activity-dependent RRP augmentation occurs in the absence of RIM1/2, only when Munc13-1 levels are extremely low or completely abolished. Consistent with this, elimination of RIM1/2 alone, a condition in which Munc13-1 levels are reduced but not absent, did not result in high-frequency stimulation-mediated RRP augmentation (Fig. $7 B$; RIM1/2 $\Delta$ Cre + Scramble: $0.89 \pm 0.03, n=31 / 4 ; \quad \mathrm{RIM} 1 / 2$ Cre + Scramble: $1.07 \pm 0.08, n=29 / 4$, $p=0.854$, Kruskal-Wallis test). Hence, RRP augmentation requires the absence of Munc13-1 but does not rely on the proximal localization of SVs to the AZ membrane by RIM1/2.

Which molecule underlies activity-dependent RRP size augmentation in the absence of Munc13-1? In hippocampal neurons, Munc13-2 is expressed at low, but detectable, levels and is responsible for the remaining synaptic transmission in Munc13-1-deficient glutamatergic neurons (Rosenmund et al., 2002). The brain-specific isoform of Munc13-2 (bMunc13-2), in particular, has been postulated to participate in augmentation (Lipstein et al., 2012). To examine whether Munc13-2 is indeed responsible for RRP augmentation, we used Munc132-deficient neurons (Munc13-2 KO) and reduced Munc13-1 expression levels by shRNA-mediated KD $\left(40 \times 10^{5} \mathrm{IU}\right)$ and examined activity-dependent RRP augmentation (Fig. 7C,D). We found that even with Munc13-1 KD, which revealed high-frequency stimulationdependent RRP augmentation in control and RIM1/2 cDKO neurons, no RRP augmentation was observed in the absence of Munc13-2 (Fig. 7D; Scramble: 0.88 $\pm 0.06, n=25 / 2$; Munc13-1 KD: $0.99 \pm 0.11, n=15 / 2, p=0.7$, Mann-Whitney test). These data demonstrate that (1) Munc13-2 does indeed mediate RRP augmentation, (2) the absence of Munc13-1 is necessary to unmask RRP augmentation, and (3) RIM1/2 is not necessary for Munc13-2's priming activity or contribution to RRP augmentation.

\section{Discussion}

Many proteins in the AZ work in concert to efficiently transduce presynaptic APs into neurotransmitter release. To understand how the AZ mediates presynaptic function, it is crucial to consider individual proteins in conjunction with their interaction partners. Here, we explored the extent to which RIM individually plays direct roles in SV localization and neurotransmission, versus its secondary roles through interactions with Munc13-1. We find that loss of RIM1/2 causes two effects on ultrastructure: impaired AZ-proximal localization of SVs and reduced SV docking. The docking deficit, in the absence of RIM, likely stems from a lack of activation and stabilization of Munc13-1. However, reduction of AZ-proximal SVs, which did not occur in Munc13-1 KO, reflects RIM's independent role in SV localization. Similar to the RIM's docking function, elec-
B

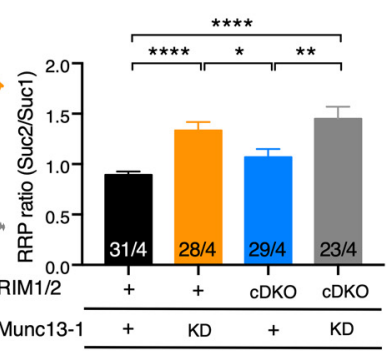

D

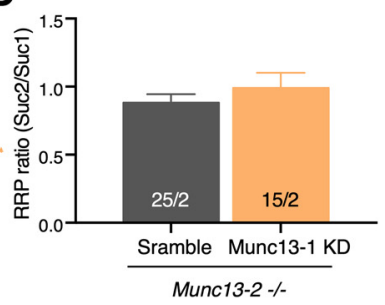

Figure 7. The regulation of RRP augmentation in the absence of RIM and Munc13-1. $\boldsymbol{A}$, Sample traces represent RRP augmentation protocol in RIM1/2 flox cultures infected with $\Delta$ Cre + Scramble (Scr.) shRNA (black), $\Delta$ (re $+40 \times 10^{5}$ IU Munc13-1 KD shRNA (orange), Cre + Scramble shRNA (blue), and Cre $+40 \times 10^{5}$ Munc13-1 A ( (Steste nonparametric $t$ test, followed by Mann-Whitney test, did not show view, see also the table in Extended Data Figure 7-1.

trophysiological analysis showed that priming function of RIM depends on Munc13-1. However, the role of RIM in SV localization does not affect activity-dependent RRP augmentation. This work provides a finer view of the extent to which RIM and Munc13 cooperate to achieve SV recruitment, docking, priming and fusion.

\section{Role of RIM and Munc13-1 in SV localization}

Recent advances in cryofixation techniques of neuronal tissue have emphasized the connection between synaptic ultrastructure and function, such as revealing the morphologic equivalent of SV priming as SV docking using high-pressure freezing fixation (Siksou et al., 2009; Imig et al., 2014). Therefore, we examined SV docking and distribution in high-pressure frozen synapses of RIM and/or Munc13-1-deficient synapses and achieved two important findings:

First, we show that RIM influences vesicle docking, which is consistent with the previous reports in murine synapses (Han et al., 2011; Kaeser et al., 2011; S. S. Wang et al., 2016) and in Caenorhabditis elegans (Gracheva et al., 2008). Nevertheless, the effect of RIM on SV docking is only attributable to Munc13-1 activation. This largely confirms the dogma of RIM acting upstream of Munc13-1 in SV docking (Han et al., 2011; Camacho et al., 2017).

Second, we find that RIM influences the distribution of SVs near the plasma membrane. This is different from Munc13-1 KO (Fig. 1) and Munc13-1/2-deficient synapses (Siksou et al., 2009; Imig et al., 2014), which demonstrated an enriched density of SVs near to the plasma membrane, but revealed severe docking deficits. The accumulation of SVs at AZ-proximal regions, which 
disappears with loss of RIM, demonstrates that the AZ-proximal SV localization relies on RIM, independent of Munc13-1. This finding extends the observations from cryo-electron tomographic studies in synaptosomes, where SVs connected with short protein "tethers" to the AZ membrane depend partially on RIM1 $\alpha$ (Fernández-Busnadiego et al., 2010, 2013). We believe that these findings are not affected by synapse size, as previous studies reported that RIM1/2 cDKO does not impair total SV number, PSD length, or bouton size (Kaeser et al., 2011; Acuna et al., 2016) and Munc13-1/2 DKO does not affect SV number per terminal and PSD length (Imig et al., 2014). In neuromuscular junction synapses of C. elegans, RIM homolog, Unc10, deficiency also impairs SV localization to the dense projections (Weimer et al., 2006), suggesting an evolutionarily conserved role for RIM in SV localization. While SV localization near the AZ membrane is mediated by RIM, other AZ proteins, such as Piccolo, Bassoon, Liprin- $\alpha 3$, and Liprin- $\alpha 2$, influence the long-range SV distribution by modulating early stages of AZ assembly and vesicle formation (Mukherjee et al., 2010; Spangler et al., 2013; Wong et al., 2018; Ackermann et al., 2019). Thus, AZ proteins act in a hierarchical order by determining the precise SV localization.

What is the physiological relevance of vesicle tethering? One hypothesis is that tethering brings SVs in close proximity to the plasma membrane to make SVs readily available for entrance into the RRP (Hallermann and Silver, 2013). However, when we examined activity-dependent changes in RRP size, a condition that shows RRP augmentation in Munc13-1-deficient synapses (Rosenmund et al., 2002), we find that RIM does not affect this phenomenon. This argues that the physiological role of RIM in tethering function manifests by different means. Essentially, the function of tethers depends on their molecular composition and interactions with scaffolds. Bassoon, a protein that modulates long-range vesicle localization, tethers SVs by reloading SVs into the pool (Hallermann et al., 2010). Nevertheless, the fact that RIM-Rab3 complex anchors SVs close to both $\mathrm{Ca}^{2+}$ channels and plasma membrane (Han et al., 2011; Kaeser et al., 2011; de Jong et al., 2018) may indicate that RIM's tethering function is a product of several molecular interactions. Therefore, the functional role of RIM in SV localization may be resolved by other electrophysiological measurements as well as by providing identity to the composition of tethers at the AZ.

\section{Role of RIM and Munc13 isoforms in vesicular release probability}

RIM defines the efficiency of release by recruiting $\mathrm{Ca}^{2+}$ channels (Han et al., 2011; Kaeser et al., 2011), binding to PIP2 rich membranes (de Jong et al., 2018) and interacting with Rab3 (Y. Wang et al., 1997; X. Wang et al., 2001; Fukuda, 2003; Schluter et al., 2006). In addition, in C. elegans, the interaction of Unc10 with Unc13 modulates $P_{\mathrm{vr}}$ (Zhou et al., 2013; Liu et al., 2019). However, we show that, in mammals, the effect of RIM on $P_{\mathrm{vr}}$ is independent from Munc13-1. Then what causes loss of $P_{\mathrm{vr}}$ in Munc13-1 KO neurons? While previous work has demonstrated that Munc13 KD impairs $\mathrm{Ca}^{2+}$ entry in hippocampal mass culture (Calloway et al., 2015), recent work in Munc13-1/2 DKO autaptic neurons shows no effect on presynaptic $\mathrm{Ca}^{2+}$ signal (Brockmann et al., 2020). As our data show that Munc13-1 reduction does not have a major effect on vesicular release probability, our findings are consistent with a lack of Munc13-1 effect on $\mathrm{Ca}^{2+}$ influx. Since only the total absence of Munc13-1, but not varying Munc13-1 levels, impairs $P_{\mathrm{vr}}$, we presume that priming with the alternative Munc13 isoform, Munc13-2, results in low release efficiency SVs. The function of Munc13-2 is only unmasked in the complete absence of Munc13-1, and not even revealed in the case of drastically reduced Munc13-1 expression levels. Furthermore, while only Munc13-2 is responsible for activity-dependent augmentation of RRP in a RIM- and Munc13-1-independent manner, both Munc13-1 and Munc13-2 contribute to dynamic changes in release probability through short-term plasticity by lowering the energy barrier for vesicle fusion (Rosenmund et al., 2002; Basu et al., 2007). Molecularly, these functions take place by activation of regulatory domains that bind to CaM, DAG (via C1 domain), and $\mathrm{Ca}^{2+}$ (via C2B domain) (Rhee et al., 2002; Junge et al., 2004; Shin et al., 2010; Lipstein et al., 2013).

\section{Other pathways of docking/priming parallel to RIM- Munc13-1}

While the relevance of RIM-Munc13-1 interactions in SV docking and release at small central synapse is clear, alternative paths exist that allow SVs to become fusion-competent. For example, bMunc13-2 or Munc13-3 does not require RIM and is differentially expressed in specific brain regions (Augustin et al., 1999b). Our ultrastructural and physiological data from Munc13-1 KD in RIM1/2 cDKO neurons, as well as Munc13-1 KO neurons, suggest that bMunc13-2, which does not require activation via RIM, accounts for $\sim 30 \%$ of docking and $\sim 5 \%$ of priming. Moreover, in invertebrate synapses, long and short Munc13s exist that form distinct complexes with $\mathrm{AZ}$ proteins and regulate different forms of release similar to Munc13-1, -2, and -3 (Brose et al., 1995; Aravamudan et al., 1999; Richmond et al., 1999; Kohn et al., 2000; Rosenmund et al., 2002; Bohme et al., 2016; Liu et al., 2019). An interesting observation from our study is that RIM1/2 cDKO shows more severe impact on SV priming in glutamatergic autaptic neurons compared with GABAergic synapses in hippocampal mass culture (Deng et al., 2011). Although these differences could be attributed to the experimental systems, it is known that GABAergic neurons, in contrast to glutamatergic neurons, redundantly use Munc13-2 for priming (Augustin et al., 1999a; Varoqueaux et al., 2002). The synapse-type-specific AZ protein composition is also evident in other vertebrate synapses. For example, in hippocampus mossy fiber synapses, RIM expression is low, and Munc13-1 uses RIM-BP to prime SVs (Brockmann et al., 2019). Overall, it is noteworthy that some synapses are prone to use more than one mode of priming.

Understanding how different synapses encode the incoming AP pattern into release requires studying the unique transduction apparatus expressed in individual synapses. We must dissect not only the individual role of molecules in the synapse but also how these molecules work together. For instance, our experiment examining the Munc13-1 concentration dependency of priming extends the previous studies (Deng et al., 2011) by showing that RIM boosts the Munc13-1 priming function by approximately fourfold. Therefore, we require more sophisticated models than single protein, loss-of-function experiments. In this study, we aimed to investigate how synapses respond to the relative changes in expression of $\mathrm{AZ}$ components by modifying the protein expression levels and studying the isoform-specific functions. We provide the first step in characterizing the role of RIM and Munc13 in small synapses to facilitate the understanding of complex molecular functions at the AZ.

\section{References}

Ackermann F, Waites CL, Garner CC (2015) Presynaptic active zones in invertebrates and vertebrates. EMBO Rep 16:923-938. 
Ackermann F, Schink KO, Bruns C, Izsvak Z, Hamra FK, Rosenmund C, Garner CC (2019) Critical role for Piccolo in synaptic vesicle retrieval. Elife 8:e46629.

Acuna C, Liu X, Sudhof TC (2016) How to make an active zone: unexpected universal functional redundancy between RIMs and RIM-BPs. Neuron 91:792-807.

Andrews-Zwilling YS, Kawabe H, Reim K, Varoqueaux F, Brose N (2006) Binding to Rab3A-interacting molecule RIM regulates the presynaptic recruitment of Munc13-1 and ubMunc13-2. J Biol Chem 281:1972019731.

Arancillo M, Min SW, Gerber S, Münster-Wandowski A, Wu YJ, Herman M, Trimbuch T, Rah JC, Ahnert-Hilger G, Riedel D, Südhof TC, Rosenmund C (2013) Titration of Syntaxin1 in mammalian synapses reveals multiple roles in vesicle docking, priming, and release probability. J Neurosci 33:16698-16714.

Aravamudan B, Fergestad T, Davis WS, Rodesch CK, Broadie K (1999) Drosophila UNC-13 is essential for synaptic transmission. Nat Neurosci 2:965-971.

Augustin I, Rosenmund C, Sudhof TC, Brose N (1999a) Munc13-1 is essential for fusion competence of glutamatergic synaptic vesicles. Nature 400:457-461.

Augustin I, Betz A, Herrmann C, Jo T, Brose N (1999b) Differential expression of two novel Munc13 proteins in rat brain. Biochem J 337:363-371.

Basu J, Betz A, Brose N, Rosenmund C (2007) Munc13-1 C1 domain activation lowers the energy barrier for synaptic vesicle fusion. J Neurosci 27:1200-1210.

Betz A, Thakur P, Junge HJ, Ashery U, Rhee JS, Scheuss V, Rosenmund C, Rettig J, Brose N (2001) Functional interaction of the active zone proteins Munc13-1 and RIM1 in synaptic vesicle priming. Neuron 30:183-196.

Bohme MA, McCarthy AW, Grasskamp AT, Beuschel CB, Goel P, Jusyte M, Laber D, Huang S, Rey U, Petzoldt AG, Lehmann M, Gottfert F, Haghighi P, Hell SW, Owald D, Dickman D, Sigrist SJ, Walter AM (2019) Rapid active zone remodeling consolidates presynaptic potentiation. Nat Commun 10:1085

Bohme MA, Beis C, Reddy-Alla S, Reynolds E, Mampell MM, Grasskamp AT, Lutzkendorf J, Bergeron DD, Driller JH, Babikir H, Gottfert F, Robinson IM, O'Kane CJ, Hell SW, Wahl MC, Stelzl U, Loll B, Walter AM, Sigrist SJ (2016) Active zone scaffolds differentially accumulate Unc13 isoforms to tune $\mathrm{Ca}(2+)$ channel-vesicle coupling. Nat Neurosci 19:1311-1320.

Brockmann MM, Maglione M, Willmes CG, Stumpf A, Bouazza BA, Velasquez LM, Grauel MK, Beed P, Lehmann M, Gimber N, Schmoranzer J, Sigrist SJ, Rosenmund C, Schmitz D (2019) RIM-BP2 primes synaptic vesicles via recruitment of Munc13-1 at hippocampal mossy fiber synapses. Elife 8:e43243.

Brockmann MM, Zarebidaki F, Camacho M, Grauel MK, Trimbuch T, Sudhof TC, Rosenmund C (2020) A trio of active zone proteins comprised of RIM-BPs, RIMs, and Munc13s governs neurotransmitter release. Cell Rep 32:107960.

Brose N, Hofmann K, Hata Y, Sudhof TC (1995) Mammalian homologues of Caenorhabditis elegans unc-13 gene define novel family of C2-domain proteins. J Biol Chem 270:25273-25280.

Calloway N, Gouzer G, Xue M, Ryan TA (2015) The active-zone protein Munc13 controls the use-dependence of presynaptic voltage-gated calcium channels. Elife 4:e07728.

Camacho M, Basu J, Trimbuch T, Chang S, Pulido-Lozano C, Chang SS, Duluvova I, Abo-Rady M, Rizo J, Rosenmund C (2017) Heterodimerization of Munc13 C2A domain with RIM regulates synaptic vesicle docking and priming. Nat Commun 8:15293.

de Jong AP, Roggero CM, Ho MR, Wong MY, Brautigam CA, Rizo J, Kaeser PS (2018) RIM C2B domains target presynaptic active zone functions to PIP2-containing membranes. Neuron 98:335-349.e337.

Deng L, Kaeser PS, Xu W, Sudhof TC (2011) RIM proteins activate vesicle priming by reversing autoinhibitory homodimerization of Munc13. Neuron 69:317-331.

Dulubova I, Lou X, Lu J, Huryeva I, Alam A, Schneggenburger R, Sudhof TC, Rizo J (2005) A Munc13/RIM/Rab3 tripartite complex: from priming to plasticity? EMBO J 24:2839-2850.

Fernández-Busnadiego R, Zuber B, Maurer UE, Cyrklaff M, Baumeister W, Lucic V (2010) Quantitative analysis of the native presynaptic cytomatrix by cryoelectron tomography. J Cell Biol 188:145-156.
Fernández-Busnadiego R, Asano S, Oprisoreanu AM, Sakata E, Doengi M, Kochovski Z, Zürner M, Stein V, Schoch S, Baumeister W, Lucić V (2013) Cryo-electron tomography reveals a critical role of RIMlalpha in synaptic vesicle tethering. J Cell Biol 201:725-740.

Fukuda M (2003) Distinct Rab binding specificity of Rim1, Rim2, rabphilin, and Noc2: identification of a critical determinant of Rab3A/Rab27A recognition by Rim2. J Biol Chem 278:15373-15380.

Gracheva EO, Hadwiger G, Nonet ML, Richmond JE (2008) Direct interactions between C. elegans RAB-3 and Rim provide a mechanism to target vesicles to the presynaptic density. Neurosci Lett 444:137-142.

Graf ER, Valakh V, Wright CM, Wu C, Liu Z, Zhang YQ, DiAntonio A (2012) RIM promotes calcium channel accumulation at active zones of the Drosophila neuromuscular junction. J Neurosci 32:16586-16596.

Gundelfinger ED, Kessels MM, Qualmann B (2003) Temporal and spatial coordination of exocytosis and endocytosis. Nat Rev Mol Cell Biol 4:127139.

Hallermann S, Silver RA (2013) Sustaining rapid vesicular release at active zones: potential roles for vesicle tethering. Trends Neurosci 36:185-194.

Hallermann S, Fejtova A, Schmidt H, Weyhersmuller A, Silver RA, Gundelfinger ED, Eilers J (2010) Bassoon speeds vesicle reloading at a central excitatory synapse. Neuron 68:710-723.

Han Y, Kaeser PS, Sudhof TC, Schneggenburger R (2011) RIM determines $\mathrm{Ca}(2)+$ channel density and vesicle docking at the presynaptic active zone. Neuron 69:304-316.

Han Y, Babai N, Kaeser P, Sudhof TC, Schneggenburger R (2015) RIM1 and RIM2 redundantly determine $\mathrm{Ca}^{2+}$ channel density and readily releasable pool size at a large hindbrain synapse. J Neurophysiol 113:255-263.

Hibino H, Pironkova R, Onwumere O, Vologodskaia M, Hudspeth AJ, Lesage F (2002) RIM binding proteins (RBPs) couple Rab3-interacting molecules (RIMs) to voltage-gated $\mathrm{Ca}(2+)$ channels. Neuron 34:411423.

Hirano M, Takada Y, Wong CF, Yamaguchi K, Kotani H, Kurokawa T, Mori MX, Snutch TP, Ronjat M, De Waard M, Mori Y (2017) C-terminal splice variants of $\mathrm{P} / \mathrm{Q}$-type $\mathrm{Ca}(2+)$ channel CaV2.1 alpha1 subunits are differentially regulated by Rab3-interacting molecule proteins. J Biol Chem 292:9365-9381.

Imig C, Min SW, Krinner S, Arancillo M, Rosenmund C, Sudhof TC, Rhee J, Brose N, Cooper BH (2014) The morphological and molecular nature of synaptic vesicle priming at presynaptic active zones. Neuron 84:416-431.

Junge HJ, Rhee JS, Jahn O, Varoqueaux F, Spiess J, Waxham MN, Rosenmund C, Brose N (2004) Calmodulin and Munc13 form a $\mathrm{Ca}^{2+}$ sensor/effector complex that controls short-term synaptic plasticity. Cell 118:389-401.

Kaeser PS, Kwon HB, Chiu CQ, Deng L, Castillo PE, Sudhof TC (2008) RIMlalpha and RIMlbeta are synthesized from distinct promoters of the RIM1 gene to mediate differential but overlapping synaptic functions. J Neurosci 28:13435-13447.

Kaeser PS, Deng L, Wang Y, Dulubova I, Liu X, Rizo J, Sudhof TC (2011) RIM proteins tether $\mathrm{Ca}^{2+}$ channels to presynaptic active zones via a direct PDZ-domain interaction. Cell 144:282-295.

Kaeser PS, Deng L, Fan M, Sudhof TC (2012) RIM genes differentially contribute to organizing presynaptic release sites. Proc Natl Acad Sci USA 109:11830-11835.

Kiyonaka S, Wakamori M, Miki T, Uriu Y, Nonaka M, Bito H, Beedle AM, Mori E, Hara Y, De Waard M, Kanagawa M, Itakura M, Takahashi M, Campbell KP, Mori Y (2007) RIM1 confers sustained activity and neurotransmitter vesicle anchoring to presynaptic $\mathrm{Ca}^{2+}$ channels. Nat Neurosci 10:691-701.

Kohn RE, Duerr JS, McManus JR, Duke A, Rakow TL, Maruyama H, Moulder G, Maruyama IN, Barstead RJ, Rand JB (2000) Expression of multiple UNC-13 proteins in the Caenorhabditis elegans nervous system. Mol Biol Cell 11:3441-3452.

Lipstein N, Schaks S, Dimova K, Kalkhof S, Ihling C, Kolbel K, Ashery U, Rhee J, Brose N, Sinz A, Jahn O (2012) Nonconserved Ca(2+)/calmodulin binding sites in Muncl3s differentially control synaptic short-term plasticity. Mol Cell Biol 32:4628-4641.

Lipstein N, Sakaba T, Cooper BH, Lin KH, Strenzke N, Ashery U, Rhee JS, Taschenberger H, Neher E, Brose N (2013) Dynamic control of synaptic vesicle replenishment and short-term plasticity by $\mathrm{Ca}(2+)$-calmodulinMunc13-1 signaling. Neuron 79:82-96.

Liu H, Li L, Nedelcu D, Hall Q, Zhou L, Wang W, Yu Y, Kaplan JM, Hu Z (2019) Heterodimerization of UNC-13/RIM regulates synaptic vesicle release probability but not priming in C. elegans. Elife 8:e40585. 
Mukherjee K, Yang X, Gerber SH, Kwon HB, Ho A, Castillo PE, Liu X, Sudhof TC (2010) Piccolo and bassoon maintain synaptic vesicle clustering without directly participating in vesicle exocytosis. Proc Natl Acad Sci USA 107:6504-6509.

Rhee JS, Betz A, Pyott S, Reim K, Varoqueaux F, Augustin I, Hesse D, Sudhof TC, Takahashi M, Rosenmund C, Brose N (2002) Beta phorbol ester- and diacylglycerol-induced augmentation of transmitter release is mediated by Munc13s and not by PKCs. Cell 108:121-133.

Richmond JE, Davis WS, Jorgensen EM (1999) UNC-13 is required for synaptic vesicle fusion in C. elegans. Nat Neurosci 2:959-964.

Rosenmund C, Stevens CF (1996) Definition of the readily releasable pool of vesicles at hippocampal synapses. Neuron 16:1197-1207.

Rosenmund C, Sigler A, Augustin I, Reim K, Brose N, Rhee JS (2002) Differential control of vesicle priming and short-term plasticity by Munc13 isoforms. Neuron 33:411-424.

Sakamoto H, Ariyoshi T, Kimpara N, Sugao K, Taiko I, Takikawa K, Asanuma D, Namiki S, Hirose K (2018) Synaptic weight set by Munc13-1 supramolecular assemblies. Nat Neurosci 21:41-49.

Schluter OM, Basu J, Sudhof TC, Rosenmund C (2006) Rab3 superprimes synaptic vesicles for release: implications for short-term synaptic plasticity. J Neurosci 26:1239-1246.

Schoch S, Gundelfinger ED (2006) Molecular organization of the presynaptic active zone. Cell Tissue Res 326:379-391.

Shin OH, Lu J, Rhee JS, Tomchick DR, Pang ZP, Wojcik SM, CamachoPerez M, Brose N, Machius M, Rizo J, Rosenmund C, Südhof TC (2010) Munc13 C2B domain is an activity-dependent $\mathrm{Ca}^{2+}$ regulator of synaptic exocytosis. Nat Struct Mol Biol 17:280-288.

Siksou L, Varoqueaux F, Pascual O, Triller A, Brose N, Marty S (2009) A common molecular basis for membrane docking and functional priming of synaptic vesicles. Eur J Neurosci 30:49-56.

Spangler SA, Schmitz SK, Kevenaar JT, de Graaff E, de Wit H, Demmers J, Toonen RF, Hoogenraad CC (2013) Liprin-alpha 2 promotes the presynaptic recruitment and turnover of RIM1/CASK to facilitate synaptic transmission. J Cell Biol 201:915-928.

Sudhof TC (2012) The presynaptic active zone. Neuron 75:11-25.

Varoqueaux F, Sigler A, Rhee JS, Brose N, Enk C, Reim K, Rosenmund C (2002) Total arrest of spontaneous and evoked synaptic transmission but normal synaptogenesis in the absence of Munc13-mediated vesicle priming. Proc Natl Acad Sci USA 99:9037-9042.

Verhage M, Sorensen JB (2008) Vesicle docking in regulated exocytosis. Traffic 9:1414-1424.

Wang SS, Held RG, Wong MY, Liu C, Karakhanyan A, Kaeser PS (2016) Fusion competent synaptic vesicles persist upon active zone disruption and loss of vesicle docking. Neuron 91:777-791.

Wang X, Hu B, Zimmermann B, Kilimann MW (2001) Rim1 and rabphilin3 bind Rab3-GTP by composite determinants partially related through N-terminal alpha -helix motifs. J Biol Chem 276:32480-32488.

Wang Y, Okamoto M, Schmitz F, Hofmann K, Sudhof TC (1997) Rim is a putative Rab3 effector in regulating synaptic-vesicle fusion. Nature 388:593-598.

Watanabe S, Rost BR, Camacho-Perez M, Davis MW, Sohl-Kielczynski B, Rosenmund C, Jorgensen EM (2013) Ultrafast endocytosis at mouse hippocampal synapses. Nature 504:242-247.

Weimer RM, Gracheva EO, Meyrignac O, Miller KG, Richmond JE, Bessereau JL (2006) UNC-13 and UNC-10/rim localize synaptic vesicles to specific membrane domains. J Neurosci 26:8040-8047.

Wong MY, Liu CL, Wang SS, Roquas AC, Fowler SC, Kaeser PS (2018) Liprin-alpha 3 controls vesicle docking and exocytosis at the active zone of hippocampal synapses. Proc Natl Acad Sci USA 115:2234-2239.

Wu X, Cai Q, Shen Z, Chen X, Zeng M, Du S, Zhang M (2019) RIM and RIM-BP form presynaptic active-zone-like condensates via phase separation. Mol Cell 73:971-984.e975.

Zhou K, Stawicki TM, Goncharov A, Jin Y (2013) Position of UNC-13 in the active zone regulates synaptic vesicle release probability and release kinetics. Elife 2:e01180 